\title{
球簇上的相对迹公式方法
}

献给冯克勤教授 80 华诞

蔡立 ${ }^{*}$, 许宾 $^{2}$

1. 清华大学丘成桐数学科学中心, 北京 100084 ;

2. 四川大学数学学院, 成都 610064

E-mail: lcai@mail.tsinghua.edu.cn, binxu@scu.edu.cn

收稿日期: 2020-12-30；接受日期: 2021-04-25; 网络出版日期: 2021-09-22；＊通信作者

国家自然科学基金 (批准号: 11971254 和 11501382) 资助项目

摘要 本文给出关于整体域上球簇 (spherical variety) 的相对迹公式 (relative trace formula) 方法的 一般框架, 并且将其应用于 Sakellaridis 和 Venkatesh 提出的关于球簇上周期积分 (period integral) 的 猜想. 这一方法对于数论研究专家是熟知的但 (至少对于我们而言) 缺乏文献. 本文的框架基于最近 Beuzart-Plessis 等 (2019) 引入的 (对于数域的) 分离谱技术, 从而避免了直接进行精细谱展开的困难. 该技术在函数域情形也可实现.

关键词 球簇 相对迹公式 $L$ - 函数 周期积分

MSC (2020) 主题分类 $11 \mathrm{~F} 70,11 \mathrm{~F} 72$

\section{Sakellaridis-Venkatesh 猜想}

令 $F$ 是一个整体域, 并且令 $G$ 是 $F$ 上的一个约化群. 记 $[G]=G(F) \backslash G(\mathbb{A})$ 是 $G$ 的自守商 (automorphic quotient), 并且 $\mathcal{A}([G])$ 是 $G(\mathbb{A})$ 上的全体自守形式空间. 令 $\pi \hookrightarrow \mathcal{A}([G])$ 是 $G(\mathbb{A})$ 上的一 个尖自守表示. 对于 $G$ 的一个子群 $H$, 考虑 $H$ 上 $\pi$ 的周期积分

$$
P_{H}(\varphi)=\int_{[H]} \varphi(h) d h, \quad \varphi \in \pi .
$$

假设 $H$ 的极大分裂环面是平凡的, 则上述积分绝对收玫, 且给出 $H(\mathbb{A})$ - 不变空间 $\operatorname{Hom}_{H(\mathbb{A})}(\pi, \mathbb{C})$ 中的 一个元素. (对于一般的自守形式, 我们需要通过一些正则化操作 (regularization) 使得周期积分收敛, 可参见文献 [1].) 
周期积分往往与 $L$ - 函数特殊值相关联 (例如, 利用 zeta 积分来表示 Rankin-Selberg $L$ - 函数). 事实 上, Sakellaridis 和 Venkatesh ${ }^{[2]}$ 推测, 对于一大类上述的群 $(G, H)$, 至少当 $\pi$ 满足 $\operatorname{dim}_{\mathbb{C}} \operatorname{Hom}_{H(\mathbb{A})}(\pi, \mathbb{C})$ $\leqslant 1$ 的条件时, (1.1) 就存在一个整体 - 局部分解. 更精确地, 记 $\bar{\pi}$ 是 $\pi$ 的复共轭, 他们猜测周期积分

$$
\varphi_{1} \otimes \varphi_{2} \in \pi \otimes \bar{\pi} \mapsto P_{H}\left(\varphi_{1}\right) P_{H}\left(\overline{\varphi_{2}}\right)
$$

构成的 $\operatorname{Hom}_{H(\mathbb{A})^{2}}(\pi \otimes \bar{\pi}, \mathbb{C})$ 中的双线性型, 分解为 $L$ - 函数的特殊值以及某些局部周期积分的 Euler 乘积. 这里, 对于 $F$ 的每个位 $v$, 这些局部周期积分应是从 Hilbert 空间 $L^{2}\left((H \backslash G)\left(F_{v}\right)\right)$ 的 Plancherel 分解得到.

如果 $F$ 上的一个 $G$ - 簇 $X$ 是正规的, 并且 (在 $\bar{F}$ 上) 存在 $G$ 的一个含 Zariski 稠密轨道的 Borel 子群, 则称它为一个球簇 (spherical variety). 从表示论的观点看, 一个仿射 $G$ - 簇 $X$ 是球簇当且仅当 $G$ - 模 $\bar{F}[X]$ 是无重 (multiplicity-free) 的 (在代数表示的范畴里). 考虑一对约化群 $(G, H)$, 其中 $H$ 是 $G$ 的闭子群. 称 $(G, H)$ 是球型对 (spherical pair), 如果它们的几何商 $X=H \backslash G$ 是一个球簇, 这里 $G$ 右作用在 $X$ 上. 这样的一对约化群非常适合用于构造周期积分. 以下列举几个球型对的例子.

- 对称 (symmetric) 情形: 如果 $H$ 是 $G$ 在某一对合 (involution) 下的不动点, 则称 $(G, H)$ 是对称 型对. 所有对称型对都是球型对. 特别地, 考虑对 $(G \times G, G)$, 其中 $G$ 对角嵌入 $G \times G$. 这是在通常迹 公式中遇到的情形.

- Whittaker 情形: $(G, N)$, 其中 $N$ 是 $G$ 的一个极大幂么子群.

- Rankin-Selberg 情形: $\left(\mathrm{GL}_{n} \times \mathrm{GL}_{n+1}, \mathrm{GL}_{n}\right)$, 其中 $\mathrm{GL}_{n}$ 对角嵌入到 $\mathrm{GL}_{n} \times \mathrm{GL}_{n+1}$.

- Gan-Gross-Prasad 情形: 令 $W_{n+1}$ 是一个关于二次域扩张 $E / F$ 的 $n+1$ 维 Hermite 空间, 并且 令 $W_{n}$ 是 $W_{n+1}$ 的一个非退化的余维数是 1 的 Hermite 空间. 此时考虑 $\left(\mathrm{U}\left(W_{n}\right) \times \mathrm{U}\left(W_{n+1}\right), \mathrm{U}\left(W_{n}\right)\right)$, 其中 $\mathrm{U}\left(W_{n}\right)$ 对角嵌入到 $\mathrm{U}\left(W_{n}\right) \times \mathrm{U}\left(W_{n+1}\right)$.

接下来依照文献 $[2$, 第 16-17 节] 来介绍 Sakellaridis-Venkatesh 的猜想. 首先考虑局部猜想. 令 $F$ 是一个 $p$ - 进 (adic) 域. 令 $G$ 是 $F$ 上的一个分裂约化群, 并且 $X$ 是 $F$ 上的一个 $G$ - 球簇. 假设在 $X(F)$ 上存在一个 $G(F)$ - 不变测度. 记 $L^{2}(X(F))$ 为关于这个测度的平方可积函数空间.

这里的局部猜想关心的是 $L^{2}(X(F))$ 上 $G(F)$ 上右正则作用诱导的西表示的 Plancherel 分解. Sakellaridis-Venkatesh 猜测这一分解由所谓的 $G$ 上的 $X$ - 可辨 (distinguished) Arthur 参量给出. 令 $W_{F}$ 是 $F$ 的 Weil 群, 而 $L_{F}$ 是如下定义的 $F$ 的 Langlands 群:

$$
L_{F}= \begin{cases}W_{F}, & \text { 如果 } F \text { 是 Archimedes 的, } \\ W_{F} \times \mathrm{SL}_{2}(\mathbb{C}), & \text { 如果 } F \text { 是非 Archimedes 的. }\end{cases}
$$

令 $G^{\vee}$ 是 $G$ 的对偶群. 一个局部 Arthur 参量是指一个如下同态:

$$
\psi: L_{F} \times \mathrm{SL}_{2}(\mathbb{C}) \longrightarrow G^{\vee}
$$

并且要求它在 $L_{F}$ 上的限制有界, 以及在 $\mathrm{SL}_{2}(\mathbb{C})$ 上的限制是代数的. 给定一个 Arthur 参量, 它与以 下态射的复合:

$$
L_{F} \rightarrow L_{F} \times \mathrm{SL}_{2}(\mathbb{C}), \quad w \mapsto\left(w,\left(\begin{array}{cc}
|w|^{1 / 2} & 0 \\
0 & |w|^{-1 / 2}
\end{array}\right)\right)
$$

定义了一个 $L$ - 参量, 称为其对应 $L$ - 参量. 人们猜测 (参见文献 [3]) 对于每个 Arthur 参量 $[\psi]$ 的 $G^{\vee}$ 共轭类, 可以 “自然地” 对应一个 $G(F)$ 的西表示 (等价类) 的有限集合, 即所谓的 $[\psi]$ 的 Arthur 表示 包 (Arthur packet). 它包含了其对应 $L$ - 参量的 $L$ - 表示包 ( $L$-packet). 
根据球簇的分类, 从 $X$ 出发, 可定义 (参见文献 $[2$, 第 2 节])

- 一个 $\mathbb{C}$ 上的对偶群 $G_{X}^{\vee}$;

- 一个同态 $G_{X}^{\vee} \times \mathrm{SL}_{2}(\mathbb{C}) \rightarrow G^{\vee}$.

称一个 Arthur 参量

$$
\psi: L_{F} \times \mathrm{SL}_{2}(\mathbb{C}) \longrightarrow G^{\vee}
$$

是 $X$ - 可辨 (distinguished) 的 (参见文献 [2, 第 16.2 小节]), 如果存在一个缓和 (tempered) 的 (即在 $L_{F}$ 有界) $L$ - 参量

$$
\phi: L_{F} \longrightarrow G_{X}^{\vee}
$$

给出它到 $G_{X}^{\vee} \times \mathrm{SL}_{2}(\mathbb{C})$ 的提升. 需要注意的是, 根据定义可知, 这里将 $\phi$ 视为一个 $X$ - 可辨参量的组 成部分.

猜想 1.1 (局部猜想的弱形式, 参见文献 $[2$, 猜想 16.2.2]) 存在一个 $G(F)$ 的西表示的直积分 分解

$$
L^{2}(X(F))=\int_{[\psi]}^{\oplus} \mathcal{H}_{[\psi]} \mu([\psi])
$$

其中

- $[\psi]$ 跑遍所有 $X$ - 可辨 Arthur 参量的 $G_{X^{-}}^{\vee}$ 共轭类;

- 测度 $\mu$ 等价于全体 $X$ - 可辨 Arthur 参量的 $G_{X}^{\vee}$ - 共轭类上存在的一个自然测度;

- $\mathcal{H}_{[\psi]}$ 同构于一个 $[\psi]$ 的 Arthur 包的某些不可约表示 (可能为空) 的直和.

注意到以上猜想只陈述了一个表示来自于 $L^{2}(X(F))$ 的 Plancherel 分解的必要条件.

接下来, 令 $H$ 是一点 $x_{0} \in X(F)$ 的稳定子群, 并且假设 $H$ 是约化的. 对于几乎所有出现在 $L^{2}(X(F))$ 的 Plancherel 分解中的不可约西表示 $\pi$, 我们将通过上述猜想构造一个 $\pi \otimes \bar{\pi}$ 上的 “典范 的” $H(F)^{2}$ - 不变的双线性配对. 它可以将上述局部猜想与之后将要讨论的整体推测联系起来.

这一构造依赖于一个对于上述 Plancherel 测度 $\mu$ 的仔细选取 (参见文献 [2, 第 17.3 小节]). 注意 到我们有一个 $G^{\vee}$ 的 $X$ - 可辨 Arthur 参量与 $G_{X}^{\vee}$ 的缓和 $L$ - 参量之间的双射, 从而可将 $\mu$ 视作一个全 体缓和 $L$ - 参量 $\phi: L_{F} \rightarrow G_{X}^{\vee}$ 的 $G_{X^{-}}^{\vee}$ 共轭类上的测度. 假设 $G_{X}^{\vee}$ 对应到 $F$ 上一个分裂群, 记为 $G_{X}$. 固定一个 $G_{X}(F)$ 上的 Haar 测度. 考虑 $G_{X}(F)^{2}$ - 表示的 Plancherel 分解

$$
L^{2}\left(G_{X}(F)\right)=\int_{[\phi]}^{\oplus} \mathcal{H}_{[\phi]} d \mu([\phi]),
$$

其中对于每个 $L$ - 参量的共轭类 $[\phi]$, 有

$$
\mathcal{H}_{[\phi]}=\bigoplus_{\pi} \pi \otimes \bar{\pi}
$$

且 $\pi$ 在与 $[\phi]$ 对应的 $L$ - 表示包里. 人们期待存在唯一的测度 $\mu$ 使得在每个 $\pi \otimes \bar{\pi}$ 上的 Hilbert 空间 结构都由 Hilbert-Schmidt 范数的整倍数给出, 且它在每个 $L$ - 表示包中存在一个不用乘整数的表示的 意义下是最小的.

令 $\mathcal{S}(X(F)) \subset C^{\infty}(X(F))$ 为 $X(F)$ 上全体 Schwartz 函数. 群 $G(F)$ 在空间 $C^{\infty}(X(F))$ 和 $\mathcal{S}(X(F))$ 上同时有作用. 进一步, $G(F)$ - 不变双线性配对

$$
\mathcal{S}(X(F)) \times C^{\infty}(X(F)) \rightarrow \mathbb{C}, \quad(f, g) \mapsto \int_{X(F)} f(x) g(x) d \mu_{X}(x)
$$


诱导了 $G(F)$ - 模之间的一个同构

$$
\mathcal{S}(X(F))^{*} \cong C^{\infty}(X(F)) .
$$

对于 $G(F)$ 上任何一个不可约光滑可容许 (admissible) 表示 $\pi$, 记

$$
\mathcal{S}(X(F))_{\pi}:=\mathcal{S}(X(F)) / \mathcal{N}_{\pi}, \quad \mathcal{N}_{\pi}:=\bigcap_{f \in \operatorname{Hom}_{G(F)}(\mathcal{S}(X(F)), \pi)} \operatorname{ker} f
$$

是 $\mathcal{S}(X(F))$ 的极大 $\pi$ - 迷向商 (maximal $\pi$-isotypic quotient).

根据 Gelfand-Kostyuchenko 方法 (参见文献 [4, 第 3.3 小节]), 对于 (关于测度 $\mu$ ) 几乎所有的 $\pi$, 分解 $(1.2)$ 决定了一个 $G(F)$ - 不变半正定 Hermite 型

$$
\langle\langle\cdot, \cdot\rangle\rangle_{\pi}: \mathcal{S}(X(F)) \times \mathcal{S}(X(F)) \rightarrow \mathbb{C}
$$

这个 Hermite 型通过映射 $\mathcal{S}(X(F)) \rightarrow \mathcal{S}(X(F))_{\pi}$ 分解, 且满足

$$
\int_{X(F)} f_{1}(x) \overline{f_{2}(x)} d x=\int_{[\psi]} \sum_{\pi \in[\psi]}\left\langle\left\langle f_{1}, f_{2}\right\rangle\right\rangle_{\pi} d \mu([\psi]), \quad \forall f_{1}, f_{2} \in \mathcal{S}(X(F)) .
$$

注意到, 由文献 $[2$, 第 6.1 小节] 知, 存在典范同构

$$
\mathcal{S}(X(F))_{\pi} \cong V(\pi) \otimes \pi, \quad V(\pi):=\operatorname{Hom}_{G(F)}(\mathcal{S}(X(F)), \pi)^{*} .
$$

注意到有典范同构 $\overline{\mathcal{S}(X(F))_{\pi}} \cong \mathcal{S}(X(F))_{\bar{\pi}}$, Hermite 型 $\langle\langle\cdot, \cdot\rangle\rangle_{\pi}$ 诱导了 $G(F)$ - 不变双线性型

$$
\langle\langle\cdot, \cdot\rangle\rangle_{\pi}: \mathcal{S}(X(F))_{\pi} \otimes \mathcal{S}(X(F))_{\bar{\pi}} \rightarrow \mathbb{C} .
$$

以下引理的证明是直接的.

引理 1.1 任意 $\pi \otimes \bar{\pi}$ 上的非退化 $G(F)$ - 不变双线性型 $(\cdot, \cdot)$ 诱导了如下同构:

$$
\begin{aligned}
& \operatorname{Hom}_{G^{2}(F)}\left(\mathcal{S}(X(F))_{\pi} \otimes \mathcal{S}(X(F))_{\bar{\pi}}, \pi \otimes \bar{\pi}\right) \stackrel{\sim}{\longrightarrow} \operatorname{Hom}_{G(F)}\left(\mathcal{S}(X(F))_{\pi} \otimes \mathcal{S}(X(F))_{\bar{\pi}}, \mathbb{C}\right), \\
& p \longmapsto(\cdot, \cdot) \circ p .
\end{aligned}
$$

固定住 $\bar{\pi} \otimes \pi$ 上的一个非退化 $G(F)$ - 不变配对 $(\cdot, \cdot)$. 由上述引理, 令

$$
p_{(\cdot, \cdot)}: \mathcal{S}(X(F)) \otimes \mathcal{S}(X(F)) \longrightarrow \bar{\pi} \otimes \pi
$$

是唯一的 $G^{2}(F)$ - 不变映射, 满足 $(\cdot, \cdot) \circ p_{(\cdot, \cdot)}=\langle\langle\cdot, \cdot\rangle\rangle_{\bar{\pi}}$. 取对偶, 得到 $G^{2}(F)$ - 不变映射:

$$
p_{(\cdot, \cdot)}^{*}: \pi \otimes \bar{\pi} \stackrel{\sim}{\longrightarrow}(\bar{\pi} \otimes \pi)^{*} \rightarrow\left(\mathcal{S}(X(F))^{2}\right)^{*} \stackrel{\sim}{\longrightarrow} C^{\infty}(X(F))^{2} .
$$

复合以上同构, 我们得到一个 $G^{2}(F)$ - 不变映射

$$
\pi \otimes \bar{\pi} \rightarrow C^{\infty}(X(F))^{2} .
$$

进一步, 复合以下 $H^{2}(F)$ - 不变映射:

$$
\mathrm{ev}_{x_{0}, x_{0}}: C^{\infty}(X(F))^{2} \rightarrow \mathbb{C}, \quad f \mapsto f\left(x_{0}, x_{0}\right),
$$


我们得到一个双 $-H(F)$ - 不变双线性映射

$$
\alpha: \pi \otimes \bar{\pi} \stackrel{p_{(\cdot, \cdot)}^{*}}{\longrightarrow} C^{\infty}(X(F))^{2} \stackrel{\mathrm{ev}_{x_{0}, x_{0}}}{\longrightarrow} \mathbb{C} .
$$

现在考虑 Sakellaridis-Venkatesh 的整体猜测. 令 $F$ 是一个数域. 令 $G$ 是 $F$ 上的一个分裂约化群, 并且 $X$ 是一个 $F$ 上的 $G$ - 球簇. 假设 $X$ 是齐次仿射 (homogeneous affine) 的, 即 $X$ 是一个齐次 $G$ 簇, 并且 $X$ 中每一点在 $G$ 中的稳定子群都是约化的. 令 $H$ 是点 $x_{0} \in X(F)$ 的稳定子群.

令 $\nu: \pi=\bigotimes_{v}^{\prime} \pi_{v} \hookrightarrow \mathcal{A}([G])$ 是一个 $G(\mathbb{A})$ 的不可约西的尖自守表示, 这里 $\nu$ 是一个取定的 $\pi$ 在 自守函数空间上的实现. 考虑关于 $\nu$ 和 $H$ 的 $\pi$ 上的周期积分 $P_{H}$, 并且假设它收玫, 从而成为空间 $\operatorname{Hom}_{H(\mathbb{A})}(\pi, \mathbb{C})$ 中的一个元素. 相似地, 记 $\bar{\pi}$ 为 $\pi$ 的复共轭, 则 $\nu$ 诱导了嵌入

$$
\bar{\nu}: \bar{\pi} \hookrightarrow \mathcal{A}([G]), \quad \varphi \mapsto \overline{\nu(\varphi)} .
$$

我们也考虑在 $\bar{\pi}$ 上, 关于 $\bar{\nu}$ 和 $H$ 的周期积分. 考虑如下整体周期 $P_{H}$ 诱导的 $H(\mathbb{A})^{2}$ - 不变双线性 配对:

$$
P_{H}\left(\varphi_{1}\right) P_{H}\left(\varphi_{2}\right), \quad \varphi_{1} \otimes \varphi_{2} \in \pi \otimes \pi .
$$

假设 $\pi$ 满足关于 $H$ 的重数一性质, 即对于 $F$ 的每个位 $v$, 有

$$
\operatorname{dimHom}_{H\left(F_{v}\right)}\left(\pi_{v}, \mathbb{C}\right) \leqslant 1
$$

那么 $\bar{\pi}$ 也满足这个性质. 特别地, 上述整体周期的双线性配对可以分解为 $\pi_{v} \otimes \bar{\pi}_{v}$ 上的局部 $H\left(F_{v}\right)^{2}-$ 不变双线性配对 $P_{H_{v}}$ 的乘积.

我们希望考虑的基本问题是给出 $P_{H_{v}}$ 的一个纯局部表达. 一个大致的推测是, $P_{H_{v}}$ 可由上述 “典 范的” 局部配对 $\alpha$ 给出. 为叙述这个猜测, 我们在 $G(\mathbb{A})$ 上选取 Tamagawa 测度 $d x$, 并且更一般地, 对 于齐次簇的 adelic 点也采用同样方式. 在 $F$ 的每个位 $v$ 处, 正规化 $G\left(F_{v}\right)$ 上的局部测度 $d x_{v}$ 使其满 足 $d x=\prod_{v} d x_{v}$, 并且对于几乎所有 $v$ 都有 $\operatorname{Vol}\left(G\left(\mathcal{O}_{v}\right), d x_{v}\right)=1$ (详见文献 [2, 第 17.1 小节]). 特别地, 我们由此规范化了 $H(\mathbb{A})$ 上的测度, 并且对于每个 $v$ 规范化了 $X\left(F_{v}\right)$ 和 $G_{X}\left(F_{v}\right)$ 上的测度, 从而由此 规范化周期积分 $P_{H}$ 和局部周期积分 $\alpha_{v}$.

猜想 1.2 (整体猜想, 参见文献 $[2$, 猜想 17.4.1]) 令 $\nu: \pi \hookrightarrow \mathcal{A}([G])$ 是 $G(\mathbb{A})$ 的一个不可约西的 尖自守表示, 并且假设其给出了一个 $X$ - 可辨的整体 Arthur 表示包. 假设 $\pi$ 满足关于 $H$ 的重数一性 质. 在上述的规范化之下, 存在一个有理数 $q(\pi)$ 使得对于任意可局部分解的 $\varphi_{1} \otimes \varphi_{2} \in \pi \otimes \bar{\pi}$, 如下等 式成立:

$$
P_{H}\left(\varphi_{1}\right) P_{H}\left(\varphi_{2}\right)=q(\pi) \cdot \prod_{v} \alpha_{v}\left(\varphi_{1, v}, \varphi_{2, v}\right) .
$$

注 1.1 上述整体猜想是 Gan-Gross-Prasad 情形 Ichino-Ikeda 猜想 (参见文献 [5] 或者第 3 节) 的推广. 以上关于 $\pi$ 给出了一个整体的 $X$ - 可辨 Arthur 表示包这一条件可看作 Ichino-Ikeda 猜想中 “缓和” (temperd) 假设的类比.

上述 Euler 乘积需要通过 $L$ - 函数来解释. 取可局部分解的 $\varphi_{1} \otimes \varphi_{2} \in \pi \otimes \bar{\pi}$, 则存在一个足够大的 位的有限集合 $S$, 它包括所有的分歧位 (对于 $G 、 H 、 \pi 、 \varphi_{1}$ 和 $\varphi_{2}$ 而言), 使得我们有以下的分解:

$$
P_{H}\left(\varphi_{1}\right) P_{H}\left(\varphi_{2}\right)=q(\pi) \cdot L_{X}^{(S)}(\pi) \cdot \prod_{v \in S} \alpha_{v}\left(\varphi_{1, v}, \varphi_{2, v}\right)
$$


这里, 形式上 $L_{X}^{(S)}(\pi)=\prod_{v \notin S} L_{X}\left(\pi_{v}\right)$, 其中 $L_{X}\left(\pi_{v}\right)$ 是某个关于 $\pi_{v}$ 的 $L$ - 函数的特殊值 (参见文献 $[2$, (17.11)]), 使得在所有的不在 $S$ 中的 $v$ 处都有 $L_{X}\left(\pi_{v}\right)=\alpha_{v}\left(\varphi_{1, v}, \varphi_{2, v}\right)$. 特别地, 如果 $q(\pi) \neq 0$, 那么整 体周期积分 $P_{H}$ 在 $\pi$ 上的限制是非零的当且仅当对于每个 $v \in S$ 有 $\alpha_{v} \neq 0$ 且 $L_{X}^{(S)}(\pi) \neq 0$.

\section{2 相对迹公式方法}

本节给出应用相对迹公式方法证明整体猜想 1.2 的一般框架. 假定需要考虑关于齐次球簇 $X=$ $H \backslash G$ 的整体猜想. 假设存在另一个约化群 $G_{1}$ 以及 $G_{1}$ - 球簇 $X_{1}=H_{1} \backslash G_{1}$, 使得对于某个 $G_{1}$ 的子群 $H_{1}^{\prime}$, 有如下两个相等的范畴商:

$$
X / / H=X_{1} / / H_{1}^{\prime} .
$$

那么, 相对迹公式方法能被用来比较 $X$ 上 $H^{2}$ - 不变的 Schwartz 分布 (distribution) 与 $X_{1}^{\prime}$ 上 $\left(H_{1}, H_{1}^{\prime}\right)$ 不变的 Schwartz 分布, 从而可以将关于球簇 $X=H \backslash G$ 的整体猜想归结到一类 $G_{1}(\mathbb{A})$ 的自守表示关 于 $H_{1}$ 和 $H_{1}^{\prime}$ 的周期积分的分解公式. 若这个分解公式是已知的 (如一些已知的 $L$ - 函数积分表示), 则 可以由此得到 $X$ 的整体猜想 (精确叙述见定理 2.4).

\section{1 一个关于球簇的相对迹公式}

令 $F$ 是一个整体域. 令 $G$ 是 $F$ 上的一个约化群, 并且 $X$ 是 $F$ 上的一个 $G$ - 球簇. 对于 $x \in X$ 以 及 $g \in G$, 记 $x \circ g$ 为 $g$ 在 $x$ 上的作用. 在相对迹公式方法中, 我们还要考虑 $G$ 的某一约化子群 $H^{\prime}$ 在 $X$ 上的作用. 我们有如下假设:

假设 2.1 (Quot) - $X$ 是齐次仿射的. 特别地, $X$ 是仿射的, 并且存在如下 $X$ 关于群 $H^{\prime}$ 作用的 范畴商 (态射):

$$
\text { inv }: X \longrightarrow Q
$$

- 记 $X^{\mathrm{rs}}$ 是 $X$ 的全体 $H^{\prime}$ - 正则半单点 (即它的 $H^{\prime}$ - 轨道是 Zariski 闭的且 $H^{\prime}$ - 稳定子群的维数 最小). 要求任意 $X^{\mathrm{rs}}$ 中元素的 $H^{\prime}$ - 稳定子群都平凡, 则 inv 的限制 $X^{\mathrm{rs}} \longrightarrow Q^{\mathrm{rs}}$ 是 $X^{\mathrm{rs}}$ 关于 $H^{\prime}-$ 作用 的几何商. 特别地, 它诱导了嵌入

$$
X^{\mathrm{rs}}(F) / H^{\prime}(F) \hookrightarrow Q^{\mathrm{rs}}(F) .
$$

注 2.1 上述对于 $H^{\prime}$ - 稳定子群的条件一般而言并不成立. 尽管如此, 很多有趣的情形, 这个条 件是满足的, 如 Jacquet-Rallis 相对迹公式中所涉及的球簇 (参见之后的第 3 节).

记 $\mathcal{S}(G(\mathbb{A}))=\bigotimes_{v}^{\prime} \mathcal{S}\left(G\left(F_{v}\right)\right)$ 为 $G(\mathbb{A})$ 上的 Schwartz 函数空间. 考虑 Schwartz 分布

$$
I(\phi)=\int_{\left[H^{\prime}\right]} K_{\phi}(h) d h, \quad \phi \in \mathcal{S}(X(\mathbb{A})),
$$

其中

$$
K_{\phi}(h)=\sum_{x \in X(F)} \phi(x \circ h) .
$$

对于一般的 $\phi$, 上述 $\left[H^{\prime}\right]$ 上的积分并不收玫.

为了避开这个技术问题, 假设 $\phi=\bigotimes_{v}^{\prime} \phi_{v}$ 是正则支集 (regular supported), 即存在 $F$ 的某个位 $v$ 使得 $\phi_{v} \in \mathcal{S}\left(X\left(F_{v}\right)\right)$ 的支集包含于 $X^{\mathrm{rs}}\left(F_{v}\right)$. 对于这样的函数, $I(\phi)$ 可被展开为 (正则) 轨道积分的求 
和

$$
I(\phi)=\sum_{a \in Q^{\mathrm{rs}}(F)} \mathcal{O}(a, \phi)
$$

这里的轨道积分定义为

$$
\mathcal{O}(a, \phi)=\prod_{v} \mathcal{O}\left(a, \phi_{v}\right)
$$

其中对于每个 $v$,

$$
\mathcal{O}\left(\cdot, \phi_{v}\right) \in C^{\infty}\left(Q^{\mathrm{rs}}\left(F_{v}\right)\right)
$$

是 $X$ 关于 $H^{\prime}$ 的轨道积分沿着嵌入 $X^{\mathrm{rs}}\left(F_{v}\right) / H^{\prime}\left(F_{v}\right) \hookrightarrow Q^{\mathrm{rs}}\left(F_{v}\right)$ 的零扩张

$$
\mathcal{O}\left(a, \phi_{v}\right)= \begin{cases}\int_{H^{\prime}\left(F_{v}\right)} \phi_{v}\left(x_{v} \circ h\right) d h, & x_{v} \in X^{\mathrm{rs}}\left(F_{v}\right), \quad \operatorname{inv}\left(x_{v}\right)=a, \\ 0, & \text { 其他情形. }\end{cases}
$$

以上被称为 $I(\phi)$ 的几何展开.

注 2.2 如果考虑 Jacquet-Rallis 相对迹公式, 则可以证明上述关于 $\phi$ 的支集条件对于缓和表示 情形不造成影响 (参见之后的第 3.2.1 小节中第一段). 而对于一般的 $\phi$, 也可以通过正则化奇异轨道 的轨道积分来得到 $I(\phi)$ 的几何展开 (参见文献 $[6]$ ).

接下来考虑 Schwartz 分布 $I(\cdot)$ 的谱展开. 首先考虑 $G(\mathbb{A})$ 上的 Schwartz 函数与 $X(\mathbb{A})$ 上的 Schwartz 函数之间的一个对应关系. 固定 $x_{0} \in X(F)$, 并且记 $H$ 为 $x_{0}$ 的 $G$-稳定子群. 由假设 2.1 知, $H$ 也是一个约化群. 考虑映射

$$
G(F) \longrightarrow X(F), \quad g \mapsto x_{0} \circ g
$$

它诱导了一个 $G(\mathbb{A})$ - 不变态射

$$
\mathcal{S}(G(\mathbb{A})) \longrightarrow \mathcal{S}(X(\mathbb{A})), \quad f \mapsto \phi_{f}
$$

其中 $\phi_{f}$ 的支集包含于 $x_{0}$ 的 $G(\mathbb{A})$ - 轨道中, 并且对于任意 $x=x_{0} \circ g$, 有如下取值:

$$
\phi_{f}(x)=\int_{H(\mathbb{A})} f(h g) d h .
$$

不难看出

$$
K_{\phi_{f}}(h)=\int_{[H]} K_{f}\left(h^{\prime}, h\right) d h^{\prime}
$$

其中

$$
K_{f}\left(h^{\prime}, h\right)=\sum_{x \in G(F)} f\left(h^{\prime-1} x h\right) .
$$

记 $L^{2}([G])$ 为 $G(\mathbb{A})$ 上全体满足如下条件的光滑函数 $\varphi$ 的 $L^{2}$ - 完备化:

- 对于每个 $\gamma \in G(F)$ 及 $g \in G(\mathbb{A})$, 有 $\varphi(\gamma g)=\varphi(g)$;

- 在 $G(F) \backslash G(\mathbb{A})$ 上, $|\varphi|^{2}$ 是可积的,

则 $G(\mathbb{A})$ 右正则作用于 $L^{2}([G])$, 记作 $R$. 对于任意 $f \in \mathcal{S}(G(\mathbb{A}))$, 记

$$
R(f)=\int_{G(\mathbb{A})} f(g) R(g) d g
$$


为右正则作用诱导的算子, 则 $R(f)$ 是一个积分算子, 其积分核函数为 $(2.3)$ 中的 $K_{f}$.

为了绕开来自群中心的收敛障碍, 我们作如下假设:

假设 2.2 (Center) 群 $G 、 H$ 和 $H^{\prime}$ 的中心的极大分裂环面都是平凡的.

我们期望 $G(\mathbb{A})$ 上的 Schwartz 分布 $I(f)=I\left(\phi_{f}\right)$ 关于 $L^{2}([G])$ 的谱分解有良好表现. 然而, 类似 于之前的几何展开, 这类谱展开通常不易得到. 为了绕开这个技术困难, 考虑拟尖的 (quasi-cuspidal) $f \in \mathcal{S}(G(\mathbb{A}))$, 即 $R(f)$ 在 $L^{2}([G])$ 中的像包含于全体尖表示形成的谱 $L_{\text {cusp }}^{2}([G])$ (参见文献 [7, 定 义 3.2$])$.

对于这些拟尖的 $f, I(f)$ 可以展开成

$$
I(f)=\sum_{\pi} I_{\pi}(f)
$$

其中 $\pi$ 跑遍 $G(\mathbb{A})$ 上所有的尖自守表示, 且

$$
I_{\pi}(f)=\int_{\left[H^{\prime}\right]} \int_{[H]} K_{f, \pi}\left(h^{\prime}, h\right) d h d h^{\prime},
$$

这里, $K_{f, \pi}$ 是 $R(f)$ 到 $\pi$ 上的限制算子的核函数, 即

$$
K_{f, \pi}\left(h^{\prime}, h\right)=\sum_{\varphi \in \mathrm{OB}(\pi)} R(f) \varphi\left(h^{\prime}\right) \overline{\varphi(h)},
$$

其中 $\mathrm{OB}(\pi)$ 是 $\pi$ 关于 $L^{2}([G])$ 上 Petersson 内积的一组正规正交基.

类似于几何展开, 我们期望 Schwartz 分布 $I_{\pi}$ 可写成局部泛函的 Euler 积. 为此, 我们作如下假设:

假设 2.3 (Multi) Hom- 空间 $\operatorname{Hom}_{H(\mathbb{A}) \times H^{\prime}(\mathbb{A})}(\pi \otimes \bar{\pi}, \mathbb{C})$ 维数不大于 1 .

对于每个 $v$, 在 $\pi_{v}$ 上固定一个不变的 Hermite 配对 $\langle\cdot, \cdot\rangle_{v}$ 使得它们的乘积 $\prod_{v}\langle\cdot, \cdot\rangle_{v}$ 是 $L^{2}([G])$ 上 的 Petersson 内积, 则在每个 $v$ 处, 由一个不变双线性配对

$$
\alpha_{v} \in \operatorname{Hom}_{H\left(F_{v}\right) \times H^{\prime}\left(F_{v}\right)}\left(\pi_{v} \otimes \bar{\pi}_{v}, \mathbb{C}\right)
$$

可如下构造一个相对局部特征 $I_{\pi_{v}}$ :

$$
I_{\pi_{v}}\left(f_{v}\right)=\sum_{\varphi} \alpha_{v}\left(\pi_{v}\left(f_{v}\right) \varphi, \varphi\right), \quad f_{v} \in \mathcal{S}\left(G\left(F_{v}\right)\right),
$$

其中 $\varphi$ 跑遍 $\pi_{v}$ 的一个关于 $\langle\cdot, \cdot\rangle_{v}$ 正交基. 从而由假设 2.3 可知存在一个分解

$$
I_{\pi}(f)=q(\pi) \cdot \prod_{v} I_{\pi_{v}}\left(f_{v}\right), \quad f=\bigotimes_{v}^{\prime} f_{v} \in \mathcal{S}(G(\mathbb{A})),
$$

其中 $q(\pi) \in \mathbb{C}$ 是一个常量.

如果 $I_{\pi_{v}}\left(f_{v}\right) \neq 0$, 则称 $f_{v} \in \mathcal{S}\left(G\left(F_{v}\right)\right)$ 是一个关于 $\pi_{v}$ 的实验函数. 同时, 称 $\phi_{v} \in \mathcal{S}\left(X\left(F_{v}\right)\right)$ 是 一个关于 $\pi_{v}$ 的实验函数, 如果它可以从一个 $G\left(F_{v}\right)$ 上关于 $\pi_{v}$ 实验函数通过 $(2.2)$ 得到. 类似地, 称 $f \in \mathcal{S}(G(\mathbb{A}))$ (或者 $\phi \in \mathcal{S}(X(\mathbb{A}))$ ) 是一个关于 $\pi$ 的整体实验函数, 如果在每个位 $v$ 处 $f_{v}$ (或者 $\phi_{v}$ ) 都 是关于 $\pi_{v}$ 的实验函数.

注 2.3 我们期望 $I(f)$ 有一个以相对特征 $I_{\pi}(f)$ 为项的谱展开, 其中 $\pi$ 跑遍所有 $G(\mathbb{A})$ 的自 守表示, 这里 $I_{\pi}(f)$ 是 $H(\mathbb{A}) \times H^{\prime}(\mathbb{A})$ - 不变的且通过自然态射 $\mathcal{S}(G(\mathbb{A})) \rightarrow \operatorname{End}(\pi)$ 分解. 事实上, 由 Langlands 关于 Eisenstein 级数以及 $L^{2}([G])$ 谱分解的理论, 有核函数的谱展开

$$
K_{f}=\sum_{[(M, \pi)]} K_{f,[(M, \pi)]}
$$


其中 $[(M, \pi)]$ 跑遍尖资料 (cuspidal data) 的等价类. 准确地讲, 这里 $M$ 是 $G$ 的一个标准的 Levi 子群 (事先固定住一个 $G$ 的极小抛物子群 $P_{0}$ 和它的极小 Levi 子群 $M_{0}$ ), $\pi$ 是 $M(\mathbb{A})$ 上的一个尖自守表示. 若存在一个关于 $M_{0}$ 的 Weyl 元 $w$ 以及某个西非分歧特征 $\xi: M(\mathbb{A})^{1} \backslash M(\mathbb{A}) \rightarrow S^{1}$, 满足关系

$$
\operatorname{Ad}(w) \circ M=M^{\prime}, \quad \operatorname{Ad}(w) \circ \pi=\pi^{\prime} \otimes \xi,
$$

则称 $(M, \pi)$ 与 $\left(M^{\prime}, \pi^{\prime}\right)$ 等价. 注意到 $M(\mathbb{A})^{1} \backslash M(\mathbb{A})$ 有一个实向量空间的结构. 因而全体上述特征 $\xi$ 的空间, 记作 $i \mathfrak{a}_{M}^{*}$, 也是一个实向量空间. 特别地, $K_{f, \pi}$ “包含了”一个由实向量空间 $i \mathfrak{a}_{M}^{*}$ 所参数化的 一 (连续) 族表示. 因而, 为了对于每个 $[(M, \pi)]$ 得到期望的谱展开, 我们需要交换 $K_{f,[(M, \pi)]}$ 中出现的 关于 $i \mathfrak{a}_{M}^{*}$ 的积分以及 $I(f)$ 中关于 $\left[H^{\prime}\right] \times[H]$ 的积分顺序. 在 Jacquet-Rallis 相对迹公式情形中, 对于 某个西群的内窥 (endoscopic) 尖自守表示的基变化提升, 这一谱展开已得到 (参见文献 [8]).

命题 2.1 假定假设 2.1 和 2.2 都成立, 则对于任意有正则支集的拟尖函数 $f \in \mathcal{S}(G(\mathbb{A}))$, 有如下 相对迹公式:

$$
\sum_{\pi} I_{\pi}(f)=\sum_{a} \mathcal{O}\left(a, \phi_{f}\right)
$$

这里, $\pi$ 跑遍 $G(\mathbb{A})$ 上的全体尖自守表示, $a$ 跑遍全体正则半单轨道. 若进一步假设 2.3 成立, 则上式 两边求和号中每一项都是 Euler 乘积.

\section{2 分离谱}

本小节依照文献 [7] 介绍该文献发展的分离谱技术. 在此之前, 人们一般选取超尖 (supercuspidal) 表示的矩阵系数 (参见文献 [9]) 作为拟尖函数, 从而得到一个所谓的 “简单迹公式”. 对于一般自守表 示而言 (例如, 没有超尖局部分量的表示), 这样的函数未必会成为实验函数, 从而不满足应用需求. 为 了克服此困难, 文献 [7] 构造了一类 Schwartz 函数空间上的乘子来得到谱的分离, 进而对于任意尖自 守表示都能得到拟尖实验函数.

给定 $G\left(\mathbb{A}_{f}\right)$ 的一个开紧子群 $K\left(\mathbb{A}_{f}\right.$ 是 $\mathbb{A}$ 中的有限 Adele 子环), 定义 $\mathcal{S}(G(\mathbb{A}))_{K}$ 为 $\mathcal{S}(G(\mathbb{A}))$ 中 由双边 $-K$ - 不变函数组成的 Schwartz 子代数. 这里, 乘法是由卷积给出的. 事实上 (参见定理 2.2 ), 对 于任意 $G(\mathbb{A})$ 的非 Eisenstein 的不可约可容许表示 $\pi$, 都存在 $\mathcal{S}(G(\mathbb{A}))_{K}$ 的一个乘子 $\mu_{\pi} \star$, 使得对于任 意 $f \in \mathcal{S}(G(\mathbb{A}))_{K}$, 都有 $\pi\left(\mu_{\pi} \star f\right)=\pi(f)$, 并且 $R\left(\mu_{\pi} \star f\right)$ 将 $L^{2}\left(G(F) \backslash G(\mathbb{A}) / K\right.$ ) (光滑函数空间的 $L^{2}$ 完备化) 映到 $L_{\text {cusp }}^{2}(G(F) \backslash G(\mathbb{A}) / K)$ (尖函数构成的字空间) 的 $\pi$ - 准迷向 ( $\pi$-nearly isotropic) 子空间 中. 这里,

- 一个复代数 $\mathcal{S}$ (不一定有乘法么元) 的乘子是一个复线性算子 $\mu \star: \mathcal{S} \rightarrow \mathcal{S}$, 其与左乘、右乘都 交换.

- 空间 $L_{\text {cusp }}^{2}(G(F) \backslash G(\mathbb{A}) / K)$ 的 $\pi$ - 准迷向子空间是指其中所有与 $\pi$ 准等价 (nearly equivalent) 的 不可约子表示 (K- 不变部分) 的直和; 而我们称两个自守表示 $\pi$ 与 $\pi^{\prime}$ 准等价, 记为 $\pi \sim \pi^{\prime}$, 如果它们 在几乎所有位 $v$ 上都等价.

接下来具体介绍 $\mu_{\pi}$ 的构造. 令 $G$ 是一个 $F$ 上连通的约化群. 令 $S_{G}$ 是 $F$ 中使得 $G_{v}$ 分歧的位 $v$ 的全体. 固定 $G\left(\mathbb{A}_{f}\right)$ 的一个极大紧子群 $K_{0}$, 并且固定 $G(\mathbb{A})$ 上的一个 Haar 测度 $d g=\prod_{v} d g_{v}$, 使得对 于每个不在 $S_{G}$ 的非 Archimedes 素位 $v, K_{0, v}$ 都是超特殊 (hyperspecial) 极大的且 $\operatorname{Vol}\left(K_{0, v}, d g_{v}\right)=1$.

记 $S \supset S_{G}$ 为一个 $F$ 的位的有限集合. 令 $K \subset K_{0}$ 是 $G\left(\mathbb{A}_{f}\right)$ 的一个开紧群, 其形如 $K=$ $\prod_{v \in S} K_{v} \times \prod_{v \notin S} K_{0, v}$. 令 $\pi=\bigotimes_{v}^{\prime} \pi_{v}$ 是 $G(\mathbb{A})$ 上一个不可约可容许表示, 并且假设 $\pi^{K}$ 非零. 特别 地, $\pi^{(S)}=\bigotimes_{v \notin S}^{\prime} \pi_{v}$ 是非分歧的. 称一个表示 $\pi$ 是 $(G, S)$-CAP 的, 如果存在 $G$ 的一个真抛物子群 
$P=M_{P} \cdot N_{P}\left(M_{P}\right.$ 是 $P$ 的 Levi 子群), 以及 $M_{P}(\mathbb{A})$ 的一个尖自守表示 $\sigma$, 使得在任意 $v \notin S$ 处, 非分 歧表示 $\pi_{v}$ 是局部正规化抛物诱导表示 $I_{P}^{G}\left(\sigma_{v}\right)$ 的一个子商.

对于任意 $v \notin S_{G}$, 令 $\mathcal{H}_{v}=C_{c}^{\infty}\left(K_{0, v} \backslash G\left(F_{v}\right) / K_{0, v}\right)$ 为 $G_{v}$ 的关于 $K_{0, v}$ 的球型 Hecke 代数 (spherical Hecke algebra). 对于任意与 $S$ 不交的 $F$ 的位的有限集 $T$, 令 $\mathcal{H}_{T}=\bigotimes_{v \in T} \mathcal{H}_{v}$. 由于球型 Hecke 代数 是交换代数, 任何 $\mathcal{H}_{T}$ 中元素在卷积作用下给出 $\mathcal{S}(G(\mathbb{A}))_{K}$ 上的一个乘子. 这一类型的乘子将在 $F$ 是 函数域情形下用来分离谱.

而如果 $F$ 是数域, 上述用来分离谱的乘子的构造不仅涉及上述 $\mathcal{H}_{T}$, 还会涉及某个 Archimedes 位的函数空间 (参见文献 [7, 第 2 节]). 令 $\left(X^{*}, \Delta, X_{*}, \Delta^{\vee}\right)$ 是关于 $G$ 的基准根资料 (based root datum $)$. 记 $\left(X^{*}, \Phi, X_{*}, \Phi^{\vee}\right)$ 是它诱导的根资料, 并且记对应的 Weyl 群为 $W$. 令 $\operatorname{Aut}\left(X^{*}, \Phi, X_{*}, \Phi^{\vee}\right)^{\odot}$ 是 $\operatorname{Aut}\left(X^{*}, \Phi, X_{*}, \Phi^{\vee}\right)$ 中阶数至多为 2 的元素的等价类的集合, 这里两个自同构 $\theta$ 与 $\theta^{\prime}$ 等价当且仅当存 在某个 $w \in W$ 使得 $\theta^{\prime}=w \theta$. 令 $\mathfrak{h}^{*}=X^{*} \bigotimes_{\mathbb{Z}} \mathbb{R}$. 对于任意阶至多为 2 的 $\theta \in \operatorname{Aut}\left(X^{*}, \Phi, X_{*}, \Phi^{\vee}\right)$, 记 $\mathfrak{h}_{\theta}^{*}$ (或 $\mathfrak{h}_{\theta}^{*-}$ ) 为 $\mathfrak{h}^{*}$ 上关于 $\theta$ 作用的 $(+1)$ - 特征子空间 (或 $(-1)$ - 特征子空间). 我们之后将 $\mathfrak{h}^{*}$ 中的每 个元素写成 $(\alpha, \beta)$, 其中 $\alpha \in \mathfrak{h}_{\theta}^{*}$, 并且 $\beta \in \mathfrak{h}_{\theta}^{*-}$. 记 $Y_{\theta}^{*}$ 为 $\rho+X^{*} \subset \mathfrak{h}^{*}$ 到 $\mathfrak{h}_{\theta}^{*-}$ 的投影, 其中 $\rho$ 是 $\Phi$ 中 正根之和的一半.

取 $[\theta] \in \operatorname{Aut}\left(X^{*}, \Phi, X_{*}, \Phi^{\vee}\right)^{\ominus}$, 并且令 $\mu \in \mathcal{O}\left(\mathfrak{h}_{\mathbb{C}}^{*}\right)$ 是 $\mathfrak{h}_{\mathbb{C}}^{*}$ 上的一个全纯函数. 我们有如下 3 个关于 $\mu$ 的条件 (参见文献 $[7$, 第 2.2 小节]):

(1) (快速垂直下降) 存在一个 $\mathfrak{h}_{\mathbb{C}}^{*}$ 上的 Euclid 范数 $\|\cdot\|$, 使得对于任意整数 $d \geqslant 0$ 和 $M>0$ 都有

$$
\sup _{\|\Re z\|<M}|\mu(z)|(1+\|z\|)^{d}<\infty, \quad z \in \mathfrak{h}_{\mathbb{C}}^{*} .
$$

(2) 对于每个 $\theta \in[\theta]$, 存在一个 $\mathfrak{h}_{\mathbb{C}}^{*}$ 上的 $W$ - 不变的 Euclid 范数 $\|\cdot\|$, 以及一个整数 $d \geqslant 0$, 使得对 于每个 $M>0$ 都有

$$
\sup _{(\alpha, \varpi)}|\mu(\alpha, \varpi)|(1+\|(\alpha, \varpi)\|)^{-d}<\infty
$$

其中 $(\alpha, \varpi) \in \mathfrak{h}_{\theta, \mathbb{C}}^{*} \times Y_{\theta}^{*}$ 且 $\|\Re \alpha\|<M$.

(3) 对于每个 $\theta \in[\theta]$, 除了有限多个 $\varpi \in Y_{\theta}^{*}$ 之外, $\mu$ 在 $\mathfrak{h}_{\theta, \mathbb{C}}^{*} \times\{\varpi\}$ 上取值为 0 .

分别记 $\mathcal{M}_{[\theta]}\left(\mathfrak{h}_{\mathbb{C}}^{*}\right)$ 以及 $\mathcal{M}_{[\theta]}^{\sharp}\left(\mathfrak{h}_{\mathbb{C}}^{*}\right)$ 为满足 $(1)$ 和 $(2)$ 的以及 $(1)$ 和 $(3)$ 的全纯函数空间. 我们有 $\mathcal{M}_{[\theta]}^{\sharp}\left(\mathfrak{h}_{\mathbb{C}}^{*}\right) \subset$ $\mathcal{M}_{[\theta]}\left(\mathfrak{h}_{\mathbb{C}}^{*}\right)$.

令 $G_{\mathbb{R}}$ 是 $\mathbb{R}$ 上的一个连通约化群, 则 $G_{\mathbb{R}}$ 给出一组基准根资料 $\left(X^{*}, \Delta, X_{*}, \Delta^{\vee}\right)$ 和一个等价类 $[\theta] \in \operatorname{Aut}\left(X^{*}, \Phi, X_{*}, \Phi^{\vee}\right)^{\ominus}$. 特别地, 我们有关于 $G_{\mathbb{R}}$ 的函数空间 $\mathcal{M}_{[\theta]}\left(\mathfrak{h}_{\mathbb{C}}^{*}\right)$ 和 $\mathcal{M}_{[\theta]}^{\sharp}\left(\mathfrak{h}_{\mathbb{C}}^{*}\right)$. 记 $\mathfrak{g}_{\mathbb{C}}$ 为 $G_{\mathbb{R}} \otimes_{\mathbb{R}} \mathbb{C}$ 的复 Lie 代数, 并且记 $\mathcal{Z}\left(\mathfrak{g}_{\mathbb{C}}\right)$ 为 $\mathfrak{g}_{\mathbb{C}}$ 的泛包络代数的中心. 从而有 Harish-Chandra 同构 $\mathcal{Z}\left(\mathfrak{g}_{\mathbb{C}}\right) \cong \mathbb{C}\left[\mathfrak{h}_{\mathbb{C}}^{*}\right]^{W}$. 对于 $G_{\mathbb{R}}$ 的一个不可约可容许表示 $\pi$, 记 $\chi_{\pi}: \mathcal{Z}\left(\mathfrak{g}_{\mathbb{C}}\right) \rightarrow \mathbb{C}$ 为它的无穷小 (infitesimal) 特征. 在 Harish-Chandra 同构下, 它等同于 $\mathfrak{h}_{\mathbb{C}}^{*}$ 中的一个 $W$ - 轨道. 特别地, 我们得到如下配对:

$$
\widehat{G_{\mathbb{R}}} \times \mathcal{O}\left(\mathfrak{h}_{\mathbb{C}}^{*}\right)^{W} \rightarrow \mathbb{C}, \quad(\pi, \mu) \mapsto \mu\left(\chi_{\pi}\right),
$$

其中记 $\widehat{G_{\mathbb{R}}}$ 为 $G_{\mathbb{R}}$ 的全体不可约可容许表示.

例 2.1 令 $D$ 是 $\mathbb{R}$ 上的一个四元数代数, 且 $G_{\mathbb{R}}=\mathrm{P} D^{\times}$. 群 $G_{\mathbb{R}}$ 可被视作 $\mathbb{R}$ 上的一个连通约化 群, 并且它的基变换 $G_{\mathbb{C}} \cong \mathrm{PGL}_{2}$. 取定 $G_{\mathbb{C}}$ 的 Borel 子群 $B_{\mathbb{C}}$ 为全体上三角矩阵, 并且记 $T_{\mathbb{C}}$ 为全体对 角矩阵. 
记 $e$ (或 $\left.e^{\vee}\right)$ 为 $T_{\mathbb{C}}$ 上的如下有理特征 (或余特征 (cocharacter)):

$$
e\left(\begin{array}{ll}
a & 0 \\
0 & b
\end{array}\right)=\frac{a}{b}, \quad e^{\vee}(x)=\left(\begin{array}{cc}
x & 0 \\
0 & x^{-1}
\end{array}\right),
$$

则 $G_{\mathbb{C}}$ 的基准根资料 $\left(X^{*}, \Delta, X_{*}, \Delta^{\vee}\right.$ ) (由 $B_{\mathbb{C}}$ 和 $T_{\mathbb{C}}$ 定义) 为

$$
X^{*}=\mathbb{Z} \cdot e, \quad \Delta=\{e\}, \quad X_{*}=\mathbb{Z} \cdot e^{\vee}, \quad \Delta^{\vee}=\left\{e^{\vee}\right\} .
$$

特别地, $\rho=\frac{1}{2} e$. 注意到 $T_{\mathbb{C}}$ 的 Weyl 群 $W$ 由 1 和 $w$ 生成, 其中

$$
w=\left(\begin{array}{ll}
0 & 1 \\
1 & 0
\end{array}\right),
$$

则 $w$ 通过 $e \mapsto-e$ 作用于 $X^{*}$ 上. 此外, 平凡自同构 $[1] \in \operatorname{Aut}\left(X^{*}, \Phi, X_{*}, \Phi^{\vee}\right)^{\ominus}$ 对应于包含 $G_{\mathbb{R}}$ 的 $G_{\mathbb{C}}$ 内形式 (inner form) 的等价类. 将 $e$ 视为基, 可将 $\mathfrak{h}^{*}$ 与一维实空间 $\mathbb{R}$ 等同, 并且取 $\mathfrak{h}_{\mathbb{C}}^{*}$ 上的 Euclid 范 数 $\|\cdot\|$ 为通常 $\mathbb{C}$ 上的范数, 则在这个例子里, 以上关于 $\mu \in \mathcal{O}\left(\mathfrak{h}_{\mathbb{C}}^{*}\right)$ 的 3 个条件可叙述如下:

(1) (快速垂直下降) 对于任意整数 $d \geqslant 0$ 以及 $M>0$, 有

$$
\sup _{\|\Re z\|<M}|\mu(z)|(1+\|z\|)^{d}<\infty, \quad z \in \mathfrak{h}_{\mathbb{C}}^{*} ;
$$

(2) 存在一个 $d \geqslant 0$ 使得

$$
\sup _{\varpi \in \rho+X^{*}}|\mu(\varpi)|(1+\|\varpi\|)^{-d}<\infty ;
$$

(3) 对于几乎所有 $\varpi \in \rho+X^{*}, \mu(\varpi)=0$.

定理 2.1 (参见文献 [7, 定理 2.11]) 对于每个元素 $\mu \in \mathcal{M}_{[\theta]}\left(\mathfrak{h}_{\mathbb{C}}^{*}\right)^{W}$, 存在唯一的乘子 $\mu \star$ : $\mathcal{S}\left(G_{\mathbb{R}}\right) \longrightarrow \mathcal{S}\left(G_{\mathbb{R}}\right)$ 使得

$$
\pi(\mu \star f)=\mu\left(\chi_{\pi}\right) \pi(f), \quad f \in \mathcal{S}\left(G_{\mathbb{R}}\right)
$$

对于 $G_{\mathbb{R}}$ 的每一个不可约可容许表示 $\pi$ 都成立.

注 2.4 记 $\widehat{G}_{\mathbb{R}}^{\mathrm{tmp}}$ 为 $G_{\mathbb{R}}$ 上全体缓和不可约表示同构类. 赋予 $\widehat{G}_{\mathbb{R}}^{\mathrm{tmp}}$ 为 $G_{\mathbb{R}}$ 的西对偶 (unitary dual) 的 Fell 拓扑的限制拓扑. 根据 Harish-Chandra 的 Plancherel 测度公式可知, 存在一个在 $\widehat{G}_{\mathbb{R}}^{\text {tmp }}$ 上的 Borel 测度 $\mu$, 使得对于任意 $f \in \mathcal{S}\left(G_{\mathbb{R}}\right)$ 都有

$$
f(g)=\int_{\widehat{G_{\mathbb{R}}} \operatorname{tmp}} \operatorname{tr}\left(\pi(g) \pi\left(f^{\vee}\right)\right) d \mu(\pi), \quad f^{\vee}(x)=f\left(x^{-1}\right) .
$$

特别地, 对于任意 $\pi \in \widehat{G}_{\mathbb{R}}^{\mathrm{tmp}}, f \in \mathcal{S}\left(G_{\mathbb{R}}\right)$ 可由 $\pi\left(f^{\vee}\right)$ 所决定. 因此可知上述 $\mu \star$ 的唯一性.

回到整体情形. 若 $G$ 是数域 $F$ 上的一个约化群, 则定义在 $\mathbb{R}$ 上的群 $G \bigotimes_{\mathbb{Q}} \mathbb{R}$ 决定了一个基准根 资料 $\left(X^{*}, \Delta, X_{*}, \Delta^{\vee}\right)$ 以及一个等价类 $\theta \in \operatorname{Aut}\left(X^{*}, \Phi, X_{*}, \Phi^{\vee}\right)^{\varnothing}$. 特别地, 根据定理 2.1 , 我们有乘子空 间 $\mathcal{M}_{[\theta]}^{\sharp}\left(\mathfrak{h}_{\mathbb{C}}^{*}\right)$.

定理 2.2 (参见文献 [7, 定理 3.6] 和 [10, 定理 1.1]) 沿用之前的记号 $K$ 和 $S$. 假设 $\pi=\bigotimes_{v} \pi_{v}$ 是 $G(\mathbb{A})$ 上的一个不可约可容许表示且 $\pi^{(S)}$ 是非分歧的, 并且假设它不是 $(G, S)$-CAP 的, 则存在一 个与 $S$ 无交的 $F$ 的位的有限集 $T$ 以及一个乘子

$$
\mu_{\pi} \in \begin{cases}\mathcal{M}_{[\theta]}^{\sharp}\left(\mathfrak{h}_{\mathbb{C}}^{*}\right)^{W} \otimes \mathcal{H}_{T}, & \text { 若 } F \text { 是一个数域, } \\ \mathcal{H}_{T}, & \text { 若 } F \text { 是一个函数域, }\end{cases}
$$


使得对于任意 $f \in \mathcal{S}(G(\mathbb{A}))_{K}$, 都有

- $R\left(\mu_{\pi} \star f\right)$ 将 $L^{2}(G(F) \backslash G(\mathbb{A}) / K)$ 映到 $L_{\text {cusp }}^{2}(G(F) \backslash G(\mathbb{A}) / K)_{\pi}$, 这里 $L_{\text {cusp }}^{2}(G(F) \backslash G(\mathbb{A}) / K)_{\pi}$ 是 $L_{\text {cusp }}^{2}(G(F) \backslash G(\mathbb{A}) / K)$ 的 $\pi$ - 准迷向子空间.

- 若 $\pi^{\prime}=\bigotimes_{v} \pi_{v}^{\prime}$ 是 $G(\mathbb{A})$ 上的一个不可约可容许表示, 并且满足 $\pi^{\prime(S)}=\pi^{(S)}$ 且 $\chi_{\pi_{\infty}^{\prime}}=\chi_{\pi_{\infty}}$ (若 $F$ 是数域), 则

$$
\pi^{\prime}\left(\mu_{\pi} \star f\right)=\pi^{\prime}(f) .
$$

对于函数域的情形, 若 $\mu_{\pi} \in \mathcal{H}_{T}$, 则称 $T$ 为 $\mu_{\pi}$ 的支集. 类似地, 对于数域情形, 称 $T$ 与全体 Archimedes 位的并为 $\mu_{\pi}$ 的支集.

命题 2.2 令 $\pi$ 是 $G(\mathbb{A})$ 上的一个尖自守表示. 令 $K$ 是 $G\left(\mathbb{A}_{f}\right)$ 的一个开紧子群, 使得 $\pi^{K} \neq 0$. 设 $\mu_{\pi}$ 是定理 2.2 给出的一个可分离 $\pi$ 的 Schwartz 代数 $\mathcal{S}(\mathbb{A})_{K}$ 的乘子. 假定假设 2.1 和 2.2 成立, 则 若 $f=\bigotimes_{v}^{\prime} f_{v} \in \mathcal{S}(G(\mathbb{A}))_{K}$ 在某个不在 $\mu_{\pi}$ 的支集中的位 $v$ 处有正则支集，

$$
\sum_{\pi^{\prime} \sim \pi} I_{\pi^{\prime}}(f)=\sum_{a} \mathcal{O}\left(a, \phi_{\mu_{\pi} \star f}\right),
$$

其中 $\pi^{\prime}$ 跑遍 $G(\mathbb{A})$ 上所有的准等价于 $\pi$ 的尖自守表示, 若 $F$ 是数域, 还需满足 $\chi_{\pi_{\infty}^{\prime}}=\chi_{\pi_{\infty}}$.

\section{3 两个相对迹公式的比较}

对于 $i=1,2$, 令 $G_{i}$ 是定义在 $F$ 上的一个约化群, 并且 $X_{i}$ 是 $F$ 上的一个仿射 $G_{i^{-}}$簇. 令 $H_{i}^{\prime}$ 是 $G_{i}$ 的一个约化的子群, 并且 $H_{i}$ 是点 $x_{i} \in X_{i}$ 在 $G_{i}$ 中的稳定子. 在 (2.1) 中, 我们已经在 $\mathcal{S}\left(G_{i}(\mathbb{A})\right)$ 上定义 了关于子群 $H_{i}^{\prime}$ 和 $H_{i}$ 的 Schwartz 分布, 这里将其记作 $I_{G_{i}}(\cdot)(i=1,2)$. 假设对于 $X_{i}(i=1,2)$, 假设 2.1 和 2.2 分别成立, 从而对于有正则支集的拟尖实验函数可以分别得到两个相对迹公式. 我们希望在如 下的假设条件下比较两个相对迹公式:

假设 $2.4(\mathrm{Comp}) \quad$ 球簇 $X_{1}$ 和 $X_{2}$ 有相同的范畴商 (分别关于 $H_{1}^{\prime}$ 和 $H_{2}^{\prime}$ )

$$
Q_{1}=Q_{2}
$$

之后我们统一用 $Q$ 来记这个范畴商.

- (Langlands 函子性) 存在如下 Langlands 对偶群之间的 $L$ - 态射:

$$
\iota:{ }^{L} G_{2} \longrightarrow{ }^{L} G_{1} \text {. }
$$

对于 $F$ 的所有位 $v$ 进一步假设关于群 $G_{i}\left(F_{v}\right)(i=1,2)$ 的局部 Langlands 对应, 即存在 $G_{i}$ 的局部 $L-$ 参量的 $G_{i}^{\vee}$ 共轭类和 $G_{i}\left(F_{v}\right)$ 的局部 $L$ - 表示包的一一对应. 从而上面的 $L$ - 态射给出了一个从 $G_{2}\left(F_{v}\right)$ 的局部 $L$ - 表示包到 $G_{1}\left(F_{v}\right)$ 的局部 $L$ - 表示包的映射.

假设 2.4 的第 1 部分说明了两个球簇 $X_{1}$ 和 $X_{2}$ 的轨道可以建立联系, 从而对应的轨道积分之间也 会有类似的联系. 令 $v$ 是 $F$ 的一个位. 一般而言, 称两个正则半单元素 $x_{1} \in X_{1}^{\mathrm{rs}}\left(F_{v}\right)$ 和 $x_{2} \in X_{2}^{\mathrm{rs}}\left(F_{v}\right)$ 相互匹配 (matching), 如果 (在假设 2.4 第 1 部分的等同意义下)

$$
\operatorname{inv}_{1}\left(x_{1}\right)=\operatorname{inv}_{2}\left(x_{2}\right)
$$

这里 $\operatorname{inv}_{i}(i=1,2)$ 是对应的范畴商态射 (参见假设 2.1).

我们希望有充分多匹配的正则半单轨道. 作如下假设: 
假设 2.5 (Comp-Orbit) 任意位 $v$ 都有

$$
\operatorname{inv}_{2}\left(X_{2}^{\mathrm{rs}}\left(F_{v}\right)\right) \subset \operatorname{inv}_{1}\left(X_{1}^{\mathrm{rs}}\left(F_{v}\right)\right) .
$$

称两个函数 $\phi_{1} \in \mathcal{S}\left(X_{1}\left(F_{v}\right)\right)$ 和 $\phi_{2} \in \mathcal{S}\left(X_{2}\left(F_{v}\right)\right)$ 互为光滑匹配函数 (smooth matching), 如果对于 任意 $a \in Q^{\mathrm{rs}}\left(F_{v}\right)$, 都有

$$
\mathcal{O}\left(a, \phi_{1}\right)=\mathcal{O}\left(a, \phi_{2}\right)
$$

注 2.5 特别地, 若 $\phi_{1}$ 与 $\phi_{2}$ 光滑匹配, 则对于任意匹配的 $x_{1} \in X_{1}^{\mathrm{rs}}\left(F_{v}\right)$ 和 $x_{2} \in X_{2}^{\mathrm{rs}}\left(F_{v}\right)$, 都有

$$
\mathcal{O}\left(\operatorname{inv}_{1}\left(x_{1}\right), \phi_{1}\right)=\mathcal{O}\left(\operatorname{inv}_{2}\left(x_{2}\right), \phi_{2}\right) .
$$

若 $x_{1}$ 不与 $X_{2}^{\mathrm{rs}}\left(F_{v}\right)$ 中任何元素匹配, 则 $\phi_{1}$ 对于 $x_{1}$ 所在轨道的轨道积分必为 0 .

另一方面, 假设 2.4 第 2 部分中的 $L$ - 态射 $\iota$ 诱导出如下两类态射:

- 在每个非分歧位 $v$ 处, $\iota$ 诱导出球 Hecke 代数之间的态射

$$
\iota_{v}: \mathcal{H}\left(G_{1}\left(F_{v}\right)\right) \longrightarrow \mathcal{H}\left(G_{2}\left(F_{v}\right)\right)
$$

这里 $\mathcal{H}\left(G_{i}\left(F_{v}\right)\right)=C_{c}^{\infty}\left(G_{i}\left(\mathcal{O}_{v}\right) \backslash G_{i}\left(F_{v}\right) / G_{i}\left(\mathcal{O}_{v}\right)\right)$. 对于任意 $f \in \mathcal{H}\left(G_{1}\left(F_{v}\right)\right)$ 以及任意 $G_{2}\left(F_{v}\right)$ 的非分歧 表示 $\pi_{v}, \iota_{v}(f)$ 由如下的等式所刻画:

$$
\operatorname{tr} \pi_{v}\left(\iota_{v}(f)\right)=\operatorname{tr}\left(\iota_{v}\left(\pi_{v}\right)(f)\right) .
$$

- 在每个 Archimedes 位 $v$ 处 ( $F$ 是数域的情形), $\iota$ 诱导出如下 Lie 代数之间的态射 (参见第 2.2 小节)

$$
\mathfrak{h}_{2}^{*} / W_{2} \longrightarrow \mathfrak{h}_{1}^{*} / W_{1},
$$

以及如下全纯函数空间之间的态射:

$$
\iota_{v}: \mathcal{M}\left(\mathfrak{h}_{1, \mathbb{C}}^{*}\right)^{W_{1}} \longrightarrow \mathcal{M}\left(\mathfrak{h}_{2, \mathbb{C}}^{*}\right)^{W_{2}} .
$$

我们之后不在记号上区分 $\iota$ 和它在不同对象上诱导的态射. 总结以上两条,$\iota$ 可诱导出如下乘子之间 的态射:

$$
\iota: \mathcal{M}\left(\mathfrak{h}_{1, \mathbb{C}}^{*}\right)^{W_{1}} \otimes \mathcal{H}\left(G_{1}\right)_{T} \longrightarrow \mathcal{M}\left(\mathfrak{h}_{2, \mathbb{C}}^{*}\right)^{W_{2}} \otimes \mathcal{H}\left(G_{2}\right)_{T},
$$

这里 $T$ 是一个 $F$ 的位的有限集合, 其与任何 Archimedes 位和分歧位不交. 注意到在函数域情形, 这 里没有 $\mathcal{M}\left(\mathfrak{h}_{i, \mathbb{C}}^{*}\right)^{W_{i}}(i=1,2)$ 的部分.

为比较相对迹公式, 在假设 2.1-2.3 和比较条件 (假设 2.4 和 2.5) 下, 我们希望有如下 3 个局部分 析的基本假设成立.

假设 2.6 令 $v$ 是 $F$ 的一个位.

(1) (光滑匹配函数的存在性) 对于任意给定的 $\phi_{2} \in \mathcal{S}\left(X_{2}\left(F_{v}\right)\right.$ ) (或者 $f_{2} \in \mathcal{S}\left(G_{2}\left(F_{v}\right)\right)$ ), 都存在 $\phi_{1} \in \mathcal{S}\left(X_{1}\left(F_{v}\right)\right)$ (或者 $f_{1} \in \mathcal{S}\left(G_{1}\left(F_{v}\right)\right)$ ) 使得 $\phi_{2}$ 和 $\phi_{1}$ 互为光滑匹配函数 (或者 $\phi_{f_{2}}$ 和 $\phi_{f_{1}}$ 互为光滑匹 配函数). 反之, 对于任意给定的 $\phi_{1} \in \mathcal{S}\left(X_{1}\left(F_{v}\right)\right)$, 满足 $\mathcal{O}\left(a, \phi_{1}\right)=0$ 对于任意 $a \notin \operatorname{inv}\left(X_{2}^{\mathrm{rs}}\left(F_{v}\right)\right)$ 成立, 则存在 $\phi_{2} \in \mathcal{S}\left(X_{2}\left(F_{v}\right)\right)$ 与 $\phi_{1}$ 光滑匹配.

(2) (基本引理) 假设 $G_{1}$ 和 $G_{2}$ 在位 $v$ 都非分歧, 则在 Haar 测度的某个规范化下, 对于任意 $f_{1} \in \mathcal{H}\left(G_{1}\left(F_{v}\right)\right)$ 都有 $\phi_{f_{1}}$ 和 $\phi_{\iota_{v}\left(f_{1}\right)}$ 互为光滑匹配函数. 
(3) (局部特征关系) 对于 $i=1,2$, 存在一族 $\mathcal{S}\left(G_{i}\left(F_{v}\right)\right)$ 上的可通过映射

$$
\mathcal{S}\left(G_{i}\left(F_{v}\right)\right) \rightarrow \operatorname{End}\left(\pi_{i, v}\right)
$$

分解的 $\left(H_{i}\left(F_{v}\right) \times H_{i}^{\prime}\left(F_{v}\right)\right)$ - 不变 Schwartz 分布 $\left\{I_{\pi_{i, v}}\right\}_{\pi_{i, v}}$, 称其为局部相对特征, 并且满足如下条件:

- 空间 $\operatorname{Hom}_{H_{i}\left(F_{v}\right)}\left(\pi_{i, v}, \mathbb{C}\right) \otimes \operatorname{Hom}_{H_{i}^{\prime}\left(F_{v}\right)}\left(\pi_{i, v}, \mathbb{C}\right)$ 是非零的当且仅当 $I_{\pi_{i, v}}$ 是非零的;

- 存在 Schwartz 分布 $I_{\pi_{1, v}}$ 与 $I_{\pi_{2, v}}$ 之间的典范比较：存在常数 $\kappa_{v} \neq 0$ 使得对于任意 $f_{1} \in$ $\mathcal{S}\left(G_{1}\left(F_{v}\right)\right)$ 和 $f_{2} \in \mathcal{S}\left(G_{2}\left(F_{v}\right)\right), f_{1}$ 和 $f_{2}$ 互为光滑匹配函数当且仅当对于任意 $G_{1}\left(F_{v}\right)$ 的局部 $L$ - 表示 包 $\Pi_{1, v}$, 都有如下等式:

$$
\sum_{\pi_{1, v} \in \Pi_{1, v}} I_{\pi_{1, v}}\left(f_{1}\right)=\kappa_{v} \sum_{\pi_{2, v} \in \iota^{-1}\left(\Pi_{1, v}\right)} I_{\pi_{2, v}}\left(f_{2}\right) .
$$

进一步, 常数 $\kappa_{v}$ 满足乘积公式 $\prod_{v} \kappa_{v}=1$.

注 2.6 在对应于西群情形 Gan-Gross-Prasad 猜想的 Jacquet-Rallis 相对迹公式情形, 上述局部 特征关系对于所有缓和局部表示都成立. 可参见定理 3.1 以及之后的注记.

假定假设 2.6 成立. 令 $f_{1}=\bigotimes_{v}^{\prime} f_{1, v} \in \mathcal{S}\left(G_{1}(\mathbb{A})\right)$ 和 $f_{2}=\bigotimes_{v}^{\prime} f_{2, v} \in \mathcal{S}\left(G_{2}(\mathbb{A})\right)$ 是两个有正则支集 的 Schwartz 函数, 并且在 $F$ 的所有位, $f_{1, v}$ 和 $f_{2, v}$ 互为光滑匹配函数, 则可以得到如下结论:

- 比较 $X_{1}$ 和 $X_{2}$ 的相对迹公式的几何展开可知,

$$
I_{G_{1}}\left(f_{1}\right)=I_{G_{2}}\left(f_{2}\right) .
$$

- 由局部特征关系, 任意 $G_{1}$ 上的乘子 $\mu_{1}$ 在 Langlands 函子性的意义下保持光滑匹配函数关系, 即若 $\mu$ 是定理 2.2 中的一个乘子, 则 $\mu \star f_{1}$ 和 $\iota(\mu) \star f_{2}$ 互为光滑匹配函数. 此结论的具体证明可参见 文献 $[7$, 命题 4.8].

接下来考虑谱展开. 令 $\pi_{1}$ 是 $G_{1}(\mathbb{A})$ 的一个不可约尖自守表示. 记 $\Pi_{1}$ 是 $G_{1}(\mathbb{A})$ 的所有与 $\pi_{1}$ 准 等价的不可约尖自守表示构成的集合. 类似地, 记 $\Pi_{2}$ 是一个由 $G_{2}(\mathbb{A})$ 的不可约尖表示 $\pi_{2}$ 组成的集 合, 要求 $\pi_{2} \in \Pi_{2}$ 满足条件 $\iota\left(\pi_{2}\right)$ 和 $\pi_{1}$ 准等价, 这里 $\iota\left(\pi_{2}\right)$ 是 $\pi_{2}$ 关于 $L-$ 态射 $\iota$ 到 $G_{1}(\mathbb{A})$ 的函子性 提升.

假设 2.7 ( $\infty$-Char) 假设 $F$ 是数域. 对于 $i=1,2, \Pi_{i}$ 中所有成员 $\pi$ 在 Archimedes 位对应的特 征 $\chi_{\pi_{\infty}}$ 都相同.

注 2.7 考虑 $G_{1}=\mathrm{GL}_{n, E}$ 和 $G_{2}=\mathrm{U}\left(W_{n}\right)$ 的情形, 其中 $W_{n}$ 是关于二次扩张 $E / F$ 的某个 $n$ 维 Hermite 空间. 此时由强重数一定理, $\Pi_{1}=\left\{\pi_{1}\right\}$ 是一个单点集而 $\Pi_{2}$ 是 $G_{2}$ 上对应 $\pi_{1}$ 的一个 $L$ - 表示 包. 此情形下已知 $L$ - 表示包中所有成员在 Archimedes 位共享同一特征.

下面的命题给出了 Schwartz 分布 $I_{\pi_{1}^{\prime}}(\cdot)\left(\pi_{1}^{\prime} \in \Pi_{1}\right)$ 与 $I_{\pi_{2}^{\prime}}(\cdot)\left(\pi_{2}^{\prime} \in \Pi_{2}\right)$ 之间的关系.

命题 2.3 假设

- 对于 $\left(X_{i}, H_{i}^{\prime}\right)(i=1,2)$, 假设 2.1-2.3 分别成立;

- 比较条件 (假设 2.4 和 2.5) 和局部比较 (假设 2.6) 成立;

- 若 $F$ 是数域, 则 Archimedes 位假设 (假设 2.7) 成立, 则对于任意有正则支集并且互为光滑匹配函数的 $f_{1} \in \mathcal{S}\left(G_{1}(\mathbb{A})\right)$ 和 $f_{2} \in \mathcal{S}\left(G_{2}(\mathbb{A})\right)$, 有如下等式:

$$
\sum_{\pi_{1}^{\prime} \in \Pi_{1}} I_{\pi_{1}^{\prime}}\left(f_{1}\right)=\sum_{\pi_{2}^{\prime} \in \Pi_{2}} I_{\pi_{2}^{\prime}}\left(f_{2}\right) .
$$

证明 在互为光滑匹配函数的 $f_{1}$ 和 $f_{2}$ 上分别作用乘子 $\mu_{\pi_{1}}$ 和 $\iota\left(\mu_{\pi_{1}}\right)$ 即可证明命题 2.3 成立. 
为了接下来比较整体 Schwartz 分布的 Euler 乘积分解, 我们希望有如下关于表示包的局部 - 整 体相容性假设:

假设 2.8 (Auto) 对于每个位 $v$, 记 $\Pi_{1, v}$ 为包含 $\pi_{1, v}$ 的局部 $L$ - 表示包, 并且令 $\Pi_{2, v}=\iota_{v}^{-1}\left(\Pi_{1, v}\right)$ 是 $\Pi_{1, v}$ 在 $L$ - 态射 $\iota_{v}$ 下的原像, 则对于 $i=1,2$, 都有 $\Pi_{i}=\left\{\bigotimes_{v}^{\prime} \pi_{i, v}^{\prime} \mid \pi_{i, v}^{\prime} \in \Pi_{i, v}\right\}$.

注 2.8 考虑 $G_{2}=\mathrm{U}\left(W_{n}\right)$ 的情形, 这里 $W_{n}$ 是关于某个二次扩张 $E / F$ 的 $n$ 维 Hermite 空间, $\mathrm{U}\left(W_{n}\right)$ 是对应的酉群. 取 $G_{1}=\mathrm{GL}_{n, E}$, 并且 $\iota=\mathrm{BC}$ 是基变换态射 (参见之后的定理 3.1 第 2 部分). 取 $\pi_{0}$ 是 $G_{2}(\mathbb{A})$ 的一个不可约尖自守表示, 并且它到 $G_{1}(\mathbb{A})$ 的基变换提升 $\mathrm{BC}\left(\pi_{0}\right)$ 也是一个尖表示. 令 $\Pi$ 是 $G_{2}(\mathbb{A})$ 的所有与 $\pi_{0}$ 准等价的尖自守表示构成的集合; 而在任意位 $v$, 记 $\Pi_{v}$ 为包含 $\pi_{0, v}$ 的 局部 $L$ - 表示包. 从而有 $\Pi=t\left\{\bigotimes_{v}^{\prime} \pi_{v} \mid \pi_{v} \in \Pi_{v}\right\}$. 事实上, 任意 $\pi \in \Pi$ 的基变换 $\mathrm{BC}(\pi)$ 都准等价 于 $\mathrm{BC}\left(\pi_{0}\right)$, 从而由一般线性群的强重数一定理 (参见文献 [11]) 可知 $\mathrm{BC}(\pi) \simeq \mathrm{BC}\left(\pi_{0}\right)$, 即对于任意位 都有 $\pi_{v} \in \Pi_{v}$. 反之, 我们需要如下来自于酉群表示的内窥分类理论的结果 (参见文献 $[12,13]$ ): 令 $\pi=\bigotimes_{v}^{\prime} \pi_{v}$ 是 $G_{2}(\mathbb{A})$ 的一个可容许表示, 并且 $\mathrm{BC}(\pi)$ 是尖自守表示, 则 $\pi$ 一定是一个尖自守表示. 由 于上面等式的右边对于任意位都选取 $\pi_{v} \in \Pi_{v}$, 并且 $\pi=\mathrm{BC}\left(\pi_{0}\right)$ 是一个尖自守表示, 可知这样组成的 $\bigotimes_{v}^{\prime} \pi_{v}$ 也是一个尖自守表示, 从而包含在 $\Pi$ 中.

对于 $\left(X_{i}, H_{i}^{\prime}\right)(i=1,2)$ 假设重数一条件 (假设 2.3), 则任取 $G_{i}(\mathbb{A})$ 的不可约尖自守表示 $\pi_{i}$, 都存 在复数 $q\left(\pi_{i}\right)$ 使得有如下的 Euler 乘积分解:

$$
I_{\pi_{i}}\left(f_{i}\right)=q\left(\pi_{i}\right) \prod_{v} I_{\pi_{i, v}}\left(f_{i, v}\right), \quad f_{i}=\bigotimes_{v}^{\prime} f_{i, v},
$$

这里 $I_{\pi_{i, v}}$ 是猜想 2.6 中出现的局部相对特征 (也可参见之后的第 3.2 小节).

我们最后假设:

假设 2.9 (L-inv) 表示包 $\Pi_{i}$ 中的元素 $\pi_{i}$ 都有相同的 $q\left(\pi_{i}\right)$, 我们将其记作 $q\left(\Pi_{i}\right)$.

定理 2.3 假设对于 $\left(X_{i}, H_{i}^{\prime}\right)(i=1,2)$,

- 几何条件 (假设 2.1 和 2.2 ) 和重数一条件 (假设 2.3) 对于这两个球簇分别成立;

- 比较条件 (假设 2.4 和 2.5) 和局部比较 (假设 2.6) 成立;

- 若 $F$ 是数域, Archimedes 位条件 (假设 2.7) 成立;

- 函子性条件 (假设 2.8 和 2.9) 成立.

若存在一个有正则支集的函数 $f_{2} \in \mathcal{S}\left(G_{2}(\mathbb{A})\right)$ 使得 $\sum_{\pi_{2}^{\prime} \in \Pi_{2}} \prod_{v} I_{\pi_{2, v}^{\prime}}\left(f_{2, v}\right) \neq 0$, 则有

$$
q\left(\Pi_{1}\right)=q\left(\Pi_{2}\right) .
$$

证明 令 $f_{1}=\bigotimes_{v}^{\prime} f_{1, v}$ 和 $f_{2}=\bigotimes_{v}^{\prime} f_{2, v}$ 是两个有正则支集的函数, 并且它们互为光滑匹配函数. 由命题 2.3 , 有

$$
\sum_{\pi_{1}^{\prime} \in \Pi_{1}} I_{\pi_{1}^{\prime}}\left(f_{1}\right)=\sum_{\pi_{2}^{\prime} \in \Pi_{2}} I_{\pi_{2}^{\prime}}\left(f_{2}\right)
$$

根据假设 2.8 , 可得

$$
\sum_{\pi_{1}^{\prime} \in \Pi_{1}} I_{\pi_{1}^{\prime}}\left(f_{1}\right)=q\left(\Pi_{1}\right) \sum_{\pi_{1}^{\prime} \in \Pi_{1}} \prod_{v} I_{\pi_{1, v}^{\prime}}\left(f_{1, v}\right)=q\left(\Pi_{1}\right) \prod_{v} \sum_{\pi_{1, v}^{\prime} \in \Pi_{1, v}} I_{\pi_{1, v}^{\prime}}\left(f_{1, v}\right) .
$$

再利用局部特征关系 (猜想 2.6 的第 3 部分) 和假设 2.8 , 得到

$$
\prod_{v} \sum_{\pi_{1, v}^{\prime} \in \Pi_{1, v}} I_{\pi_{1, v}^{\prime}}\left(f_{1, v}\right)=\prod_{v} \kappa_{v} \sum_{\pi_{2, v}^{\prime} \in \Pi_{2, v}} I_{\pi_{2, v}^{\prime}}\left(f_{2, v}\right)=\sum_{\pi_{2}^{\prime} \in \Pi_{2}} \prod_{v} I_{\pi_{2, v}^{\prime}}\left(f_{2, v}\right) .
$$


从而

$$
q\left(\Pi_{1}\right) \sum_{\pi_{2}^{\prime} \in \Pi_{2}} \prod_{v} I_{\pi_{2, v}^{\prime}}\left(f_{2, v}\right)=q\left(\Pi_{2}\right) \sum_{\pi_{2}^{\prime} \in \Pi_{2}} \prod_{v} I_{\pi_{2, v}^{\prime}}\left(f_{2, v}\right)
$$

由此可得定理的结论.

现在考虑第 1 节中的整体猜想. 令 $X=H \backslash G$ 是一个齐次仿射的 $G$-球簇. 考虑 $G(\mathbb{A})$ 的一个西不 可约尖自守表示 $\pi=\bigotimes_{v}^{\prime} \pi_{v}$, 并且取定它在自守形式空间的实现 $\nu: \pi \hookrightarrow \mathcal{A}([G])$. 考虑 $\pi$ 的周期积分

$$
P_{H}(\varphi)=\int_{[H]} \varphi(\nu(h)) d h, \quad \varphi \in \pi
$$

对于每个位 $v$, 假设:

- 空间 $\operatorname{Hom}_{H\left(F_{v}\right)}\left(\pi_{v}, \mathbb{C}\right)$ 的维数小于等于 1 ;

- 局部猜想 1.1 对于 $X\left(F_{v}\right)$ 成立且 $\pi_{v} \otimes \bar{\pi}_{v}$ 上的 “典范” $H\left(F_{v}\right)^{2}$ - 不变的双线性配对 $\alpha_{v}$ 存在, 并 且构成空间 $\operatorname{Hom}_{H\left(F_{v}\right)^{2}}\left(\pi_{v} \otimes \bar{\pi}_{v}, \mathbb{C}\right)$ 的基.

在以上局部假设下, 与猜想 1.2 相同, 我们通过选取 Tamagawa 测度来规范化 $P_{H}$ 和每一个 $\alpha_{v}$, 则整 体猜想如下叙述: 存在一个有理数 $q(\pi)$, 使得对于任意纯张量尖形式 $\varphi_{1} \otimes \varphi_{2} \in \pi \otimes \bar{\pi}$,

$$
P_{H}\left(\varphi_{1}\right) P_{H}\left(\varphi_{2}\right)=q(\pi) \prod_{v} \alpha_{v}\left(\varphi_{1, v}, \varphi_{2, v}\right)
$$

总成立.

定理 2.4 考虑上述尖自守表示 $\pi$ 的整体猜想 1.2 . 假设有另一个 $G^{\prime}$ - 球簇 $X^{\prime}=H_{1}^{\prime} \backslash G^{\prime}$, 以及 $G^{\prime}$ 的约化子群 $H_{2}^{\prime}$ 使得 $(X, H)$ 和 $\left(X^{\prime}, H_{2}^{\prime}\right)$ 满足如下条件 (同定理 2.3 的假设条件, 并且取 $G_{2}=G$, $\left.G_{1}=G^{\prime}\right)$ :

- 几何条件 (假设 2.1 和 2.2 ) 和重数一条件 (假设 2.3 ) 对于这两个球簇分别成立;

- 比较条件 (假设 2.4 和 2.5) 和局部比较 (假设 2.6) 成立;

- 若 $F$ 是数域, 则 Archimedes 位条件 (假设 2.7) 成立;

- 函子性条件 (假设 2.8 和 2.9 ) 成立.

进一步假设

- 存在 $G^{\prime}(\mathbb{A})$ 上的一个尖自守表示 $\pi^{\prime}$ 使得对于假设 2.4 中的 $L$ - 态射 $\iota$, 对于几乎所有位 $v$, 有 $\iota\left(\pi_{v}\right)=\iota\left(\pi_{v}^{\prime}\right)$.

- 存在一个有正则支集的函数 $f \in \mathcal{S}(G(\mathbb{A}))$ 使得

$$
\sum_{\pi_{1}} \prod_{v} I_{\pi_{1, v}}\left(f_{v}\right) \neq 0
$$

其中 $\pi_{1}$ 跑遍所有与 $\pi$ 准等价的自守尖表示.

那么若存在有理数 $q\left(\pi^{\prime}\right)$ 使得如下 Schwartz 分布的分解成立:

$$
I_{\pi^{\prime}}\left(f^{\prime}\right)=q\left(\pi^{\prime}\right) \prod_{v} I_{\pi_{v}^{\prime}}\left(f_{v}^{\prime}\right), \quad f^{\prime}=\bigotimes_{v}^{\prime} f_{v}^{\prime} \in \mathcal{S}\left(G^{\prime}(\mathbb{A})\right),
$$

则整体猜想对于 $\pi$ 成立, 并且 $q(\pi)=q\left(\pi^{\prime}\right)$. 
证明 注意到该整体猜想等价于如下 $G(\mathbb{A})$ 上 Schwartz 分布的分解 (等价性的证明可参见文 献 $\left[14\right.$, 引理 1.7]): 取定一个 $\pi \otimes \bar{\pi}$ 上的非退化不变双线性配对 $\langle\cdot, \cdot\rangle$, 对于每个位 $v$, 取定一个 $\pi_{v} \otimes \bar{\pi}_{v}$ 上的非退化不变双线性配对 $\langle\cdot, \cdot\rangle_{v}$, 使得 $\langle\cdot, \cdot\rangle=\bigotimes_{v}^{\prime}\langle\cdot, \cdot\rangle_{v}$, 则

$$
I_{\pi}(f)=q(\pi) \prod_{v} I_{\pi_{v}}\left(f_{v}\right), \quad f=\bigotimes_{v}^{\prime} f_{v} \in \mathcal{S}(G(\mathbb{A})),
$$

这里

$$
I_{\pi}(f)=\sum_{\varphi} P_{H}(\nu(\pi(f) \varphi)) P_{H}(\bar{\nu}(\varphi)),
$$

其中 $\varphi$ 跑遍 $\pi$ 的一组正规正交基; 而在任意位 $v$, 有

$$
I_{\pi_{v}}\left(f_{v}\right)=\sum_{\varphi_{v}} \alpha_{v}\left(\pi_{v}\left(f_{v}\right) \varphi_{v}, \varphi_{v}\right)
$$

因而, 本定理是定理 2.3 的直接推论.

\section{3 例子: $\mathrm{U}_{n} \times \mathrm{U}_{n+1}$ 情形的 Gan-Gross-Prasad 猜想}

本节给出一个利用以上相对迹公式方法的完整例子. 该例子利用 Jacquet-Rallis 相对迹公式证明了 西群 $\mathrm{U}_{n} \times \mathrm{U}_{n+1}$ 情形下 Gan-Gross-Prasad 猜想的加细版本 (参见文献 [15]), 该精确版本也称为 IchinoIkeda 猜想 (参见文献 [5]). 为保证上一节中所涉及的假设都成立, 本节只考虑缓和稳定 (stable) 表示 情形, 更一般情形可参见文献 $[7,8]$. 本节涉及的主要结果是基于 Zhang ${ }^{[9,14] 、 B e u z a r t-P l e s s i s}{ }^{[16,17]}$ 、

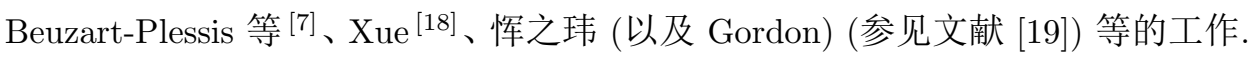

\subsection{Jacquet-Rallis 情形的球簇}

令 $E / F$ 是一个数域的二次扩张, $\left(W_{n+1},\langle\cdot, \cdot\rangle\right)$ 是 $E$ 上的一个 $n+1$ 维非退化 Hermite 空间, 并且 $W_{n}$ 是 $W_{n+1}$ 中的一个余维数为 1 的 Hermite 子空间. 记 $W_{n+1}=W_{n} \oplus E \cdot e$, 这里 $e \in W_{n+1}$ 是一个 满足 $\langle e, e\rangle=1$ 的非迷向向量.

记 $G=\mathrm{U}\left(W_{n}\right) \times \mathrm{U}\left(W_{n+1}\right)$ 以及 $H=\mathrm{U}\left(W_{n}\right)$, 这里 $H \hookrightarrow G$ 是一个对角嵌入. 我们有以下同构:

$$
X:=H \backslash G \simeq \mathrm{U}\left(W_{n+1}\right), \quad\left(g_{1}, g_{2}\right) \mapsto g_{1}^{-1} g_{2},
$$

从而 $G$ 中的 $H^{2}$ - 轨道可由 $\mathrm{U}\left(W_{n+1}\right)$ 中的 $\mathrm{U}\left(W_{n}\right)$ - 共轭类来给出. 此外, 我们还有如下的范畴商:

$$
X \longrightarrow X / / H \text {. }
$$

另一方面, 令 $G^{\prime}=\mathrm{GL}_{n, E} \times \mathrm{GL}_{n+1, E}, H_{1}=\mathrm{GL}_{n, E}$ 以及 $H_{2}=\mathrm{GL}_{n} \times \mathrm{GL}_{n+1}$. 这里取 $H_{1} \hookrightarrow G^{\prime}$ 为 对角嵌入, 并且 $H_{2} \hookrightarrow G^{\prime}$ 为对应分量的自然嵌入. 在群 $G^{\prime}$ 上, 群 $H_{1}$ 有一个左平移作用, 而群 $H_{2}$ 有 一个右平移作用. 在此情形下, 我们有如下同构:

$$
H_{1} \backslash G^{\prime} \simeq \mathrm{GL}_{n+1, E},
$$

从而 $G^{\prime}$ 中的 $\left(H_{1} \times H_{2}\right)$ - 轨道可以等同于 $\mathrm{GL}_{n+1, E}$ 中的 $H_{2}$ - 轨道. 注意到

$$
\left(H_{1} \backslash G^{\prime}\right) / H_{2} \simeq \mathrm{GL}_{n, E} \backslash \mathrm{GL}_{n+1, E} / \mathrm{GL}_{n+1} .
$$


考虑上面同构中右手边的右作用部分, 它可等同于如下的对称空间:

$$
X^{\prime}:=\mathrm{GL}_{n+1, E} / \mathrm{GL}_{n+1} \simeq S_{n+1}=\left\{s \in \mathrm{GL}_{n+1, E} \mid s \bar{s}=1\right\}, \quad g \mapsto g \bar{g}^{-1} .
$$

从而 $G^{\prime}$ 中的 $\left(H_{1} \times H_{2}\right)$ - 轨道可由 $S_{n+1}$ 中的 $\mathrm{GL}_{n^{-}}$共轭类给出. 在此情形下, 考虑范畴商

$$
X^{\prime} \longrightarrow X^{\prime} / / H_{2} \text {. }
$$

可以得到如下关于范畴商的典范同构:

$$
Q^{\prime}:=X^{\prime} / / H_{2}=X / / H=: Q .
$$

特别地, $X$ 和 $X^{\prime}$ 满足假设 2.4 中的第 1 部分.

称 $g \in \mathrm{GL}_{n+1, E}$ (关于 $\mathrm{GL}_{n, E^{-}}$共轭) 是正则半单的, 如果

$$
\left\{E_{n+1}, g \cdot E_{n+1}, \ldots, g^{n} \cdot E_{n+1}\right\}
$$

是 $\operatorname{Mat}_{n+1,1}(E)$ 的一组基, 并且

$$
\left\{E_{n+1}^{\mathrm{T}}, E_{n+1}^{\mathrm{T}} \cdot g, \ldots, E_{n+1}^{\mathrm{T}} \cdot g^{n}\right\}
$$

是 $\operatorname{Mat}_{1, n+1}(E)$ 的一组基, 这里 $E_{n+1}=\left(\begin{array}{llll}0 & \cdots & 0 & 1\end{array}\right)^{\mathrm{T}}$. 记 $\mathrm{GL}_{n+1, E}^{\mathrm{rs}} \subset \mathrm{GL}_{n+1, E}$ 为正则半单元素构成的 子集, 则类似可以记 $\mathrm{U}\left(W_{n+1}\right)^{\mathrm{rs}}=\mathrm{U}\left(W_{n+1}\right) \cap \mathrm{GL}_{n+1, E}^{\mathrm{rs}}, S_{n+1}^{\mathrm{rs}}=S_{n+1} \cap \mathrm{GL}_{n+1, E}^{\mathrm{rs}}$. 可以证明这样定义的正 则半单元和一般定义 (轨道为 Zariski 闭的且稳定子位数最小) 是吻合的. 可以证明 $g_{1}, g_{2} \in \mathrm{U}\left(W_{n+1}\right)^{\mathrm{rs}}$ (或者 $s_{1}, s_{2} \in S_{n+1}^{\mathrm{rs}}$ ) 是 $\mathrm{GL}_{n, E^{-}}$共轭的当且仅当它们是 $\mathrm{U}\left(W_{n}\right)$ - 共轭 (或者 $\mathrm{GL}_{n^{-}}$共轭) 的.

命题 3.1 以上给出的两个球簇 $X$ 和 $X^{\prime}$ 分别在群 $H$ 和 $\mathrm{GL}_{n, E}$ 的作用下满足假设 2.1. 特别地, $X^{\mathrm{rs}}$ (或者 $X^{\prime \mathrm{rs}}$ ) 在群 $H$ (或者 $\left.H_{2}\right)$ 的作用下的稳定子是平凡的.

\subsection{Jacquet-Rallis 相对迹公式}

本小节分别对于以上的西群和一般线性群情形建立相对迹公式.

\subsection{1 酉群情形}

下面继续使用上一小节的记号. 令 $f_{1} \in \mathcal{S}(G(\mathbb{A}))$ 是一个拟尖的 Schwartz 函数. 为避免收玫性的 问题, 我们进一步要求 $f_{1} \in \mathcal{S}(G(\mathbb{A}))$ 在 $X(\mathbb{A})$ 上对应的函数 $\phi_{f_{1}}=\bigotimes_{v}^{\prime} \phi_{f_{1, v}} \in \mathcal{S}(X(\mathbb{A})$ ) (参见第 2.1 小 节) 有正则的支集. 注意到文献 [9, 附录 $\mathrm{A}]$ 中的主要结果说明 $G\left(F_{v}\right)$ 的任意缓和局部表示都有带正 则支集的实验函数, 从而, 以上的要求对于本小节考虑的情形总是满足的.

取定 $G(\mathbb{A})$ 的一个不可约缓和尖自守表示 $\pi$, 并且记 $\mu_{\pi}$ 为第 2.2 小节中所定义的关于 $\pi$ 的乘子. 我们有如下 Schwartz 分布的等式:

$$
\sum_{a \in Q^{\mathrm{rs}}(F)} \mathcal{O}\left(a, \phi_{\mu_{\pi} \star f_{1}}\right)=\sum_{\pi^{\prime} \sim \pi} I_{\pi^{\prime}}\left(f_{1}\right),
$$

这里

- 等式 (3.1) 左边的 $\mathcal{O}(a, \phi)=\prod_{v} \mathcal{O}\left(a, \phi_{v}\right)$ 是关于 $a$ 的轨道积分, 其局部定义为

$$
\mathcal{O}\left(a, \phi_{v}\right)=\int_{H\left(F_{v}\right)} \phi_{v}(a \cdot h) d h ;
$$


- 等式 (3.1) 右边关于表示 $\pi^{\prime}$ 的 Schwartz 分布 $I_{\pi^{\prime}}$ 由如下积分定义:

$$
I_{\pi^{\prime}}\left(f_{1}\right)=\int_{[H]} \int_{[H]} K_{f_{1}, \pi^{\prime}}\left(h^{\prime}, h\right) d h d h^{\prime},
$$

其中的核函数 $K_{f_{1}, \pi^{\prime}}$ 由 (2.4) 给出 (在 (2.4) 中取 $H^{\prime}=H=\mathrm{U}\left(W_{n}\right)$ ).

在我们对于测试函数 $f_{1}$ 的要求下, 以上所有的求和以及积分都是收玫的.

由以上构造可知,

$$
I_{\pi}\left(f_{1}\right)=\sum_{\varphi \in \mathrm{OB}(\pi)} P_{H}\left(R\left(f_{1}\right) \varphi\right) \overline{P_{H}(\varphi)} .
$$

这也被称为关于 $\pi$ 的整体相对特征. 这里 $\mathrm{OB}(\pi)$ 是 $\pi$ 关于 Petterson 内积的一组正规正交基. 进一 步, 利用重数一定理 ${ }^{[20]}$ 可知, 存在一个 (与 $\pi$ 有关的) 常数 $q(\pi)$ 使得, 对于任意纯张量的 $\pi$ 中自守形 式 $\varphi_{1}=\bigotimes_{v}^{\prime} \varphi_{1, v}, \varphi_{2}=\bigotimes_{v}^{\prime} \varphi_{2, v}$, 有

$$
P_{H}\left(\varphi_{1}\right) \overline{P_{H}\left(\varphi_{2}\right)}=q(\pi) \cdot \prod_{v} \alpha_{v}\left(\varphi_{1, v}, \varphi_{2, v}\right)
$$

这里

$$
\alpha\left(\varphi_{1, v}, \varphi_{2, v}\right)=\int_{H\left(F_{v}\right)}\left\langle\pi_{v}(h) \varphi_{1, v}, \varphi_{2, v}\right\rangle_{v} d h,
$$

其中 $\langle\cdot, \cdot\rangle_{v}$ 来自于一个取定的整体 Petersson 内积的局部分解 $\langle\cdot, \cdot\rangle_{\mathrm{Pet}}=\prod_{v}\langle\cdot, \cdot\rangle_{v} \cdot$ 称

$$
I_{\pi_{v}}\left(f_{1, v}\right)=\sum_{\varphi_{v} \in \mathrm{OB}\left(\pi_{v}\right)} \alpha_{v}\left(\pi_{v}\left(f_{1, v}\right) \varphi_{v}, \varphi_{v}\right)
$$

为 $\pi_{v}$ 的局部相对特征, 这里 $\mathrm{OB}\left(\pi_{v}\right)$ 是 $\pi_{v}$ 关于 $\langle\cdot, \cdot\rangle_{v}$ 的一组正规正交基.

\subsection{2 一般线性群情形}

将数域扩张 $E / F$ 对应的二次特征记为 $\eta$. 取 $h=\left(h_{1}, h_{2}\right) \in H_{2}$, 对于 $n$ 是奇数或者偶数的情形, 分别记 $\eta(h)=\eta\left(\operatorname{det}\left(h_{2}\right)\right)$ 或者 $\eta(h)=\eta\left(\operatorname{det}\left(h_{1}\right)\right)$. 与之前的情形相同, 取 $f_{2} \in \mathcal{S}\left(G^{\prime}(\mathbb{A})\right)$ 是一个拟尖 的 Schwartz 函数, 并且要求它对应一个有正则支集的函数 $\phi_{f_{2}}^{\prime}=\bigotimes_{v}^{\prime} \phi_{f_{2, v}}^{\prime} \in \mathcal{S}\left(X^{\prime}(\mathbb{A})\right)$. 取定 $G^{\prime}(\mathbb{A})$ 的一个不可约缓和尖自守表示 $\Pi$, 并且记 $\mu_{\Pi}$ 为第 2.2 小节中所定义的关于 $\Pi$ 的乘子. 我们有如下 Schwartz 分布的等式:

$$
\sum_{a \in Q^{\prime \mathrm{rs}}(F)} \mathcal{O}^{\prime}\left(a, \phi_{\mu_{\Pi} \star f_{2}}^{\prime}\right)=\sum_{\Pi^{\prime} \sim \Pi} I_{\Pi^{\prime}}\left(f_{2}^{\prime}\right)
$$

这里

- 等式 (3.5) 左边的 $\mathcal{O}^{\prime}(a, \phi)=\prod_{v} \mathcal{O}^{\prime}\left(a, \phi_{v}\right)$ 是关于 $a$ 的轨道积分, 其局部定义为

$$
\mathcal{O}^{\prime}\left(a, \phi_{v}\right)=\int_{H_{2}\left(F_{v}\right)} \phi_{v}(a \cdot h) \eta(h) d h ;
$$

- 等式 (3.5) 右边关于 $\Pi^{\prime}$ 的 Schwartz 分布 $I_{\Pi^{\prime}}$ 由如下积分定义:

$$
I_{\Pi^{\prime}}\left(f_{2}\right)=\int_{\left[H_{1}\right]} \int_{\left[H_{2}\right]} K_{f_{2}, \Pi^{\prime}}\left(h^{\prime}, h\right) \eta(h) d h d h^{\prime},
$$


其中核函数 $K_{f_{2}, \Pi^{\prime}}$ 由 (2.4) 给出 (在 (2.4) 中取 $H^{\prime}=H_{1}$ 和 $H=H_{2}$ ).

注意到在以上的 Schwartz 分布中引进特征 $\eta$ 是为了将其与整体 Flicker-Rallis 周期积分联系起来 (参 见文献 $[14,21])$.

由以上构造可知,

$$
I_{\Pi}\left(f_{2}\right)=\sum_{\varphi^{\prime} \in \mathrm{OB}(\Pi)} P_{H_{1}}\left(R\left(f_{2}\right) \varphi^{\prime}\right) \overline{P_{H_{2}, \eta}\left(\varphi^{\prime}\right)}
$$

这里

$$
P_{H_{2}, \eta}\left(\varphi^{\prime}\right)=\int_{Z_{H_{2}}(\mathbb{A}) H_{2}(F) \backslash H_{2}(\mathbb{A})} \varphi^{\prime}\left(h_{2}\right) \eta\left(h_{2}\right) d h_{2}
$$

称为 Flicker-Rallis 周期积分 (我们也将 $P_{H_{1}}\left(\varphi^{\prime}\right)$ 称作 Rankin-Selberg 周期积分).

考虑如下关于局部表示 $\Pi_{v}$ 的局部相对特征 $I_{\Pi_{v}}$. 取定一个非平凡的加法特征 $\psi: F \backslash \mathbb{A} \rightarrow \mathbb{C}^{\times}$. 对 于一个 $\mathrm{GL}_{k}\left(\mathbb{A}_{E}\right)$ 的不可约西尖自守表示 $\Pi_{k}$, 考虑它的整体 Whittaker 模型 $\mathcal{W}\left(\Pi_{k}, \psi_{E}\right)$ 以及它的局部 对应 $\mathcal{W}\left(\Pi_{k, w}, \psi_{E, w}\right)\left(w\right.$ 是 $E$ 的一个位), 这里 $\psi_{E}(\cdot)=\psi\left(\frac{1}{2} \operatorname{tr}_{E / F}(\cdot)\right)$. 对于 $W_{w}, W_{w}^{\prime} \in \mathcal{W}\left(\Pi_{k, w}, \psi_{E, w}\right)$, 可以定义如下的不变内积 (参见文献 $[14,22]$ ):

$$
\vartheta_{k, w}\left(W_{w}, W_{w}^{\prime}\right)=\int_{N_{k-1}\left(E_{w}\right) \backslash \mathrm{GL}_{k-1}\left(E_{w}\right)} W_{w}\left(\left(\begin{array}{l}
h \\
1
\end{array}\right)\right) \overline{W_{w}^{\prime}\left(\left(\begin{array}{l}
h \\
1
\end{array}\right)\right)} d h,
$$

这里 $N_{r}$ 是 $\mathrm{GL}_{r}$ 中的极大标准 (上三角) 么幕子群. 记 $\vartheta_{w}=\vartheta_{n, w} \otimes \vartheta_{n+1, w}$. 从而对于 $F$ 的任意位 $v$ 以及 $G^{\prime}\left(F_{v}\right)$ 的一般 (generic) 西表示 $\Pi_{v}=\Pi_{n, v} \otimes \Pi_{n+1, v}$, 我们可以考虑 $\vartheta_{v}\left(W_{v}\right)\left(W_{v} \in \mathcal{W}\left(\Pi_{v}, \psi_{E_{v}}\right)\right)$, 这里 $\mathcal{W}\left(\Pi_{v}, \psi_{E_{v}}\right)=\mathcal{W}\left(\Pi_{n, v}, \bar{\psi}_{E_{v}}\right) \otimes \mathcal{W}\left(\Pi_{n+1, v}, \psi_{E_{v}}\right)$.

为定义 $I_{\Pi_{v}}$, 我们还需要用到局部 Flicker-Rallis 周期积分和局部 Rankin-Selberg 周期积分. 取 $W_{v} \in \mathcal{W}\left(\Pi_{v}, \psi_{E_{v}}\right)$ 和 $\varphi^{\prime} \in \Pi_{v}$, 按照如下定义局部 Flicker-Rallis 周期积分:

$$
\beta_{v}\left(W_{v}\right)=\int_{N_{n-1}(F) \backslash \mathrm{GL}_{n-1}\left(F_{v}\right)} W_{v}\left(\left(\begin{array}{c}
\epsilon_{n-1} h \\
1
\end{array}\right)\right) \eta(h) d h,
$$

这里 $\epsilon_{n-1}=\operatorname{diag}\left\{\tau^{n-1}, \tau^{n-2}, \ldots, \tau\right\}$, 其中 $\tau$ 是二次扩张 $E / F$ 的生成元 $(E=F[\tau])$. 另一方面, 取 $s \in \mathbb{C}$ 和 $W_{v} \in \mathcal{W}\left(\Pi_{v}, \psi_{E_{v}}\right)$, 按照如下定义局部 Rankin-Selberg 周期积分:

$$
\lambda_{v}\left(s, W_{v}\right)=\int_{N_{n}\left(E_{v}\right) \backslash \mathrm{GL}_{n}\left(E_{v}\right)} W_{v}(h)|\operatorname{det} h|_{E_{v}}^{s} d h .
$$

可以证明当 $\Re(s) \gg 0$ 时, 局部积分 $\lambda_{v}\left(s, W_{v}\right)$ 绝对收玫, 并且可以延拓成为整个复平面上的整函数. 结合以上, 局部相对特征 $I_{\Pi_{v}}$ 由如下表达式定义:

$$
I_{\Pi_{v}}\left(f_{2, v}\right)=\sum_{W_{v}} \frac{\lambda_{v}\left(0, \Pi_{v}\left(f_{2, v}\right) W_{v}\right) \bar{\beta}_{v}\left(W_{v}\right)}{\vartheta_{v}\left(W_{v}\right)},
$$

这里 $W_{v}$ 跑遍 $\mathcal{W}\left(\Pi_{v}, \psi_{E_{v}}\right)$ 的一组关于 (3.6) 的正交基.

\section{3 相对迹公式的比较}

\subsection{1 轨道匹配及主要局部结果}

分别取 $\delta \in \mathrm{U}\left(W_{n+1}\right)^{\mathrm{rs}}(F)$ 和 $s \in S_{n+1}^{\mathrm{rs}}(F)$. 如果 $s$ 和 $\delta$ (都看作 $\mathrm{GL}_{n+1}(E)$ 中的元素) 可由 $\mathrm{GL}_{n}(E)$ 中的元素通过共轭作用相互得到, 则称 $s$ 和 $\delta$ 相互匹配. 这等价于它们在范畴商映射下的像一致. 进 
一步, 元素之间的匹配给出了如下轨道之间的对应关系:

$$
\bigsqcup_{\mathcal{W}_{n}} \mathrm{U}\left(\mathcal{W}_{n+1}\right)^{\mathrm{rs}}(F) / \mathrm{U}\left(\mathcal{W}_{n}\right)(F) \simeq S_{n+1}^{\mathrm{rs}}(F) / \mathrm{GL}_{n}(F)
$$

这里 $\mathcal{W}_{n}$ 取遍所有的 $n$ 维 $E$-Hermite 空间的同构类, 并且对于某个固定的一维 Hermite 空间 $E \cdot e$, $\mathcal{W}_{n+1}=\mathcal{W}_{n} \oplus E \cdot e$ (参见文献 $[23$, 第 2 节 $]$ ).

取 $F$ 的一个位 $v$. 考虑函数 $\phi^{\prime} \in \mathcal{S}\left(S_{n+1}\left(F_{v}\right)\right)$ 以及

$$
\left\{\phi^{\mathcal{W}_{n}}\right\}_{\mathcal{W}_{n}} \in \bigoplus_{\mathcal{W}_{n}} \mathcal{S}\left(\mathrm{U}\left(\mathcal{W}_{n+1}\right)\left(F_{v}\right)\right)
$$

这里的求和与 (3.8) 中相同. 如果在 $\delta \in \mathrm{U}\left(W_{n+1}\right)^{\mathrm{rs}}(F)$ 和 $s \in S_{n+1}^{\mathrm{rs}}(F)$ 相互匹配时有如下等式成立:

$$
\Omega_{v}(s) \mathcal{O}\left(s, \phi^{\prime}\right)=\mathcal{O}\left(\delta, \phi^{\mathcal{W}_{n}}\right),
$$

则称 $\phi^{\prime}$ 和 $\left\{\phi^{\mathcal{W}_{n}}\right\}$ 互为光滑匹配函数, 并且记为

$$
\phi^{\prime} \leftrightarrow\left\{\phi^{\mathcal{W}_{n}}\right\}
$$

这里 $\left\{\Omega_{v}\right\}_{v}$ 是 $G^{\prime r s}\left(F_{v}\right)$ 上的一族函数, 称其为转移因子 (transfer factor) (参见文献 [9, 第 2.4 小节]), 并且要求其对于任意 $\gamma \in G^{\prime \mathrm{rs}}(F)$ 都满足乘积公式 $\prod_{v} \Omega_{v}(\gamma)=1$.

为比较相对迹公式, 我们需要如下的局部结果:

定理 3.1 - (光滑匹配 ${ }^{[18,23]}$ ) 若 $v$ 非 Archimedes 位, 则每个 $\phi^{\prime} \in \mathcal{S}\left(S_{n+1}\left(F_{v}\right)\right)$ 都和某一族 $\left\{\phi^{\mathcal{W}_{n}}\right\} \mathcal{W}_{n}$ 互为光滑匹配函数, 反之亦然. 若 $v$ 是 Archimedes 位, 则存在 $\mathcal{S}\left(S_{n+1}\left(F_{v}\right)\right)$ 的一个稠密子集 使得其中的任意函数都和某一族 $\left\{\phi^{\mathcal{W}_{n}}\right\} \mathcal{W}_{n}$ 互为光滑匹配函数, 反之亦然.

- (基本引理) 在任意非分歧位 $v$, 对于任意 $f_{1} \in \mathcal{H}\left(G\left(F_{v}\right)\right.$ ) (参见 (2.5) 以及猜想 2.6 第 2 部分), $\phi_{f_{1, v}}$ 和 $\phi_{\mathrm{BC}\left(f_{1, v}\right)}$ 互为光滑匹配函数, 这里 $\mathrm{BC}$ 是 Langlands 对偶群之间的基变换态射

$$
\mathrm{BC}:{ }^{L} \mathrm{U}\left(W_{m}\right)=\mathrm{GL}_{m}(\mathbb{C}) \rtimes W_{F_{v}} \rightarrow{ }^{L} \mathrm{GL}_{m, E}=\left(\mathrm{GL}_{n}(\mathbb{C}) \times \mathrm{GL}_{n}(\mathbb{C})\right) \rtimes W_{F_{v}},
$$

它具体由 $(g, \sigma) \mapsto\left(\left(g, J_{m}{ }^{\mathrm{T}} g J_{m}^{-1}\right), \sigma\right)$ 给出, 这里

$$
J_{m}=\left(\begin{array}{ll} 
& 1 \\
& \cdot \\
(-1)^{m-1} &
\end{array}\right) .
$$

- $\left(\right.$ 相对特征关系 $\left.{ }^{[16]}\right)$ 存在满足 $\prod_{v} \kappa_{v}=1$ 的精确常数 $\left(\kappa_{v}\right)_{v}$, 使得对于任意 $G\left(F_{v}\right)$ 的满足

$$
\operatorname{Hom}_{H\left(F_{v}\right)}\left(\pi_{v}, \mathbb{C}\right) \neq 0
$$

不可约缓和表示 $\pi_{v}$, 以及满足 $\phi_{f_{1, v}} \leftrightarrow \phi_{f_{2, v}}^{\prime}$ 的 Schwartz 函数 $f_{1, v} \in \mathcal{S}\left(G\left(F_{v}\right)\right)$ 和 $f_{2, v} \in \mathcal{S}\left(G^{\prime}\left(F_{v}\right)\right)$, 都有

$$
I_{\pi_{v}}\left(f_{1, v}\right)=\kappa_{v} \cdot I_{\mathrm{BC}\left(\pi_{v}\right)}\left(f_{2, v}\right) .
$$


注 3.1 以上定理的第 2 部分称为 Hecke 代数的基本引理, 它可以由定理的第 3 部分 (相对特征 关系) 得到 (参见文献 [24]). 它的一个特殊情形, 单位函数的基本引理, 即 $\mathbf{1}_{S_{n+1}\left(\mathcal{O}_{v}\right)} \leftrightarrow \mathbf{1}_{\mathrm{U}\left(\mathcal{W}_{n+1}\right)\left(\mathcal{O}_{v}\right)}$, 是由恽之玮 (以及 Gordon) 证明的 (参见文献 [19]). 该结果在 Beuzart-Plessis ${ }^{[17]}$ 关于相对特征关系的 证明中起到了重要作用.

注 3.2 注意到, 若特征关系 (3.10) 成立, 则可推知 $\phi_{f_{1, v}} \leftrightarrow \phi_{f_{2, v}}^{\prime}$ (参见文献 [7, 引理 4.9]). 从而 结合以上第 2 部分可知, 对于 $G\left(F_{v}\right)$ 的任意不可约缓和尖自守表示 $\pi_{v}$, 以及它的基变换 $\mathrm{BC}\left(\pi_{v}\right)$, 若 $\phi_{f_{1, v}} \leftrightarrow \phi_{f_{2, v}}^{\prime}$, 则有 $\phi_{\mu_{\pi} \star f_{1, v}} \leftrightarrow \phi_{\mu_{\mathrm{BC}(\pi)}^{\prime} \star f_{2, v}}^{\prime}$ (参见文献 [7, 命题 4.8]).

\subsection{2 比较}

现在可以比较 (3.1) 和 (3.5) 两个相对迹公式. 令 $\pi$ 是 $G(\mathbb{A})$ 的不可约缓和尖自守表示, 并且记 $\mathrm{BC}(\pi)$ 是它到 $G^{\prime}(\mathbb{A})$ 的基变换提升. 我们进一步要求 $\mathrm{BC}(\pi)$ 也是一个尖表示 (即之前假设的稳定情 形). 记 $G^{\mathcal{W}_{n}}=\mathrm{U}\left(\mathcal{W}_{n}\right) \times \mathrm{U}\left(\mathcal{W}_{n+1}\right)$, 这里 $\mathcal{W}_{n}$ 和 $\mathcal{W}_{n+1}$ 与之前的定义相同. 取拟尖 Schwartz 函数

$$
f_{1}=\left(f_{1}^{\mathcal{W}_{n}}\right)_{\mathcal{W}_{n}} \in \bigoplus_{\mathcal{W}_{n}} \mathcal{S}\left(G^{\mathcal{W}_{n}}(\mathbb{A})\right)
$$

以及 $f_{2} \in \mathcal{S}\left(G^{\prime}(\mathbb{A})\right)$, 使得 $\phi_{f_{1}}$ 和 $\phi_{f_{2}}^{\prime}$ 都有正则的支集, 并且在 $F$ 的每个位都有 $\phi_{f_{1, v}} \leftrightarrow \phi_{f_{2, v}}^{\prime}$. 由相对 迹公式 (3.1) 和 (3.5), 以及定理 3.1 的第 1 和 2 部分, 得到如下等式:

$$
\sum_{\substack{\mathcal{W}_{n} \\ \pi^{\prime} \leftrightarrow \mathcal{A}_{\text {cusp }}\left(G^{\prime} \mathcal{W}_{n}(\mathrm{~A})\right) \\ \pi^{\prime} \sim \pi}} I_{\pi^{\prime}}\left(f_{1}^{\mathcal{W}_{n}}\right)=\sum_{\substack{\Pi \hookrightarrow \mathcal{A}_{\text {cusp }\left(G^{\prime}(\mathrm{A})\right)} \\ \Pi^{\prime} \sim \mathrm{BC}(\pi)}} I_{\Pi^{\prime}}\left(f_{2}\right)=I_{\mathrm{BC}(\pi)}\left(f_{2}\right),
$$

其中最后一个等号来自 $\mathrm{GL}_{N}$ 的强重数一定理 (注意到 $\mathrm{BC}(\pi)$ 是一个尖表示). 进一步, 由 $(3.2)$ 和 (3.3), 以及 $\mathrm{U}_{n} \times \mathrm{U}_{n+1}$ 情形的局部 Gan-Gross-Prasad 猜想 (参见文献 [17,25-27]) 可知, 只有唯一一个 $n$ 维 Hermite 空间, 记作 $\mathcal{W}_{n}^{*}$, 以及唯一一个 $G^{\mathcal{W}_{n}^{*}}(\mathbb{A})$ 的尖表示 $\pi^{\prime} \sim \pi$, 使得整体相对特征 $I_{\pi^{\prime}}\left(f_{1}^{\mathcal{W}_{n}^{*}}\right)$ 非零. 从而等式 $(3.11)$ 给出

$$
I_{\pi^{\prime}}\left(f_{1}^{\mathcal{\mathcal { W } _ { n } ^ { * }}}\right)=I_{\mathrm{BC}(\pi)}\left(f_{2}\right)
$$

这里 $f_{1}^{\mathcal{W}{ }_{n}^{*}}$ 与 $f_{2}$ 和之前的选取相同.

\section{4 在加细 Gan-Gross-Prasad (Ichino-Ikeda) 猜想上的应用}

最后一小节利用等式 (3.12) 来得到 $\mathrm{U}_{n} \times \mathrm{U}_{n+1}$ 情形的加细 Gan-Gross-Prasad 猜想 (参见文 献 $[7,8,14,15]$ ), 这是 Sakellaridis-Venkatesh 的整体猜想 (猜想 1.2) 对于球簇 $X$ 的一个特例. 我们 先在本节考虑的情形叙述这个猜想. 基于之前提到的工作 $[7,14,16]$, 该猜想目前已经成为如下的定理:

定理 3.2 (参见文献 [7, 定理 1.9]) 令 $\pi$ 是 $G(\mathbb{A})$ 的一个不可约缓和尖自守表示, 并且它到 $G^{\prime}(\mathbb{A})$ 的基变换提升 $\mathrm{BC}(\pi)$ 也是一个尖表示. 则对于任意可分解自守形式 $\varphi=\bigotimes_{v}^{\prime} \varphi_{v} \in \pi$, 有如下等式成立:

$$
\frac{\left|P_{H}(\varphi)\right|^{2}}{\langle\varphi, \varphi\rangle_{\mathrm{Pet}}}=\frac{\Delta_{n+1}}{4} \cdot \frac{L\left(\frac{1}{2}, \mathrm{BC}(\pi)\right)}{L(1, \pi, \mathrm{Ad})} \prod_{v} \frac{\alpha_{v}^{\natural}\left(\varphi_{v}, \varphi_{v}\right)}{\left\langle\varphi_{v}, \varphi_{v}\right\rangle_{v}},
$$

这里

$$
\text { - } L(s, \pi, \mathrm{Ad})=L\left(s, \pi_{n}, \mathrm{Ad}\right) \cdot L\left(s, \pi_{n+1}, \mathrm{Ad}\right) \text {, 其中 } \pi=\pi_{n} \otimes \pi_{n+1} ;
$$


- $\Delta_{n+1}=\prod_{i=1}^{n+1} L\left(i, \eta_{E / F}^{i}\right)$;

- $\alpha_{v}^{\natural}(\cdot, \cdot)$ 是如下定义的规范化局部 $G\left(F_{v}\right)$ - 不变的双线性型:

$$
\alpha_{v}^{\natural}\left(\widetilde{\varphi}_{v}, \varphi_{v}\right)=\frac{L\left(1, \pi_{v}, \mathrm{Ad}\right)}{\Delta_{n+1, v} \cdot L\left(\frac{1}{2}, \mathrm{BC}\left(\pi_{v}\right)\right)} \cdot \alpha_{v}\left(\widetilde{\varphi}_{v}, \varphi_{v}\right) .
$$

类似于 (3.4), 可定义如下规范化局部相对特征:

$$
I_{\pi_{v}}^{\natural}\left(f_{v}\right)=\sum_{\varphi_{v} \in \mathrm{OB}\left(\pi_{v}\right)} \alpha_{v}^{\natural}\left(\pi_{v}\left(f_{v}\right) \varphi_{v}, \varphi_{v}\right), \quad f_{v} \in \mathcal{S}\left(G\left(F_{v}\right)\right) .
$$

定理 3.2 等价于如下的定理 (参见文献 [14, 引理 1.5]):

定理 3.3 令 $\pi$ 是 $G(\mathbb{A})$ 的一个不可约缓和尖自守表示, 并且它到 $G^{\prime}(\mathbb{A})$ 的基变换提升 $\mathrm{BC}(\pi)$ 也是一个尖表示. 则对于任意 Schwartz 函数 $f_{1}=\bigotimes_{v}^{\prime} f_{1, v} \in \mathcal{S}(G(\mathbb{A}))$, 有如下等式成立:

$$
I_{\pi}\left(f_{1}\right)=\frac{\Delta_{n+1}}{4} \cdot \frac{L\left(\frac{1}{2}, \mathrm{BC}(\pi)\right)}{L(1, \pi, \mathrm{Ad})} \cdot \prod_{v} I_{\pi_{v}}^{\natural}\left(f_{1, v}\right)=\frac{1}{4} \cdot \prod_{v} I_{\pi_{v}}\left(f_{1, v}\right) .
$$

为证明 (3.15), 只需要考虑 $\prod_{v} I_{\pi_{v}} \neq 0$ 的情形, 即上一小节中 $W_{n}=\mathcal{W}_{n}^{*}$ 的情形. 此时, 对于 $f_{2}=\bigotimes_{v}^{\prime} f_{2, v} \in \mathcal{S}\left(G^{\prime}(\mathbb{A})\right)$ 使得在所有位 $v$ 满足 $\phi_{f_{1, v}} \leftrightarrow \phi_{f_{2, v}}^{\prime}$, 我们有 (来自 (3.12))

$$
I_{\pi}\left(f_{1}\right)=I_{\mathrm{BC}(\pi)}\left(f_{2}\right) .
$$

另一方面, Jacuqet 等 [28] 和 Flicker-Rallis (参见文献 [21]) 的工作对于整体 Rankin-Selberg 周期积分 $P_{H_{1}}\left(\varphi^{\prime}\right)$ 和整体 Flicker-Rallis 周期积分 $P_{H_{2}, \eta}\left(\varphi^{\prime}\right)$ 分别给出了精确的 Euler 乘积分解, 从而给出了一个 $I_{\mathrm{BC}(\pi)}\left(f_{2}\right)$ 的精细 Euler 乘积分解 (参见文献 [14, 第 3 节]):

$$
I_{\mathrm{BC}(\pi)}\left(f_{2}\right)=\frac{1}{4} \cdot \prod_{v} I_{\mathrm{BC}\left(\pi_{v}\right)}\left(f_{2, v}\right) .
$$

根据之前的叙述, 上面的 Euler 乘积分解可以看作一个对于球簇 $X^{\prime}$ 的 Sakellaridis-Venkatesh 型公式. 从而, 由 (3.16) 以及定理 3.1 中的第 3 部分, 我们可以最终得到等式 (3.15).

致谢 冯克勤教授是我国代数数论和算术代数几何研究的开拓者, 为现代数论在中国的发展作出了巨大贡献. 本文的 两位作者早年在四川大学求学过程中, 也是通过冯克勤教授的经典中文著作《代数数论》开始了对现代数论的学习. 我 们非常荣幸能为冯克勤教授八十岁寿辰撰文, 祝冯克勤教授健康长寿.

\section{参考文献}

1 Zydor M. Periods of automorphic forms over reductive subgroups. arXiv:1903.01697, 2019

2 Sakellaridis Y, Venkatesh A. Periods and harmonic analysis on spherical varieties. Astérisque, 2017, 396: 1-296

3 Arthur J. The Endoscopic Classification of Representations: Orthogonal and Symplectic Groups. Colloquium Publications, vol. 61. Providence: Amer Math Soc, 2013

4 Li W-W. Zeta Integrals, Schwartz Spaces and Local Functional Equations. Lecture Notes in Mathematics, vol. 2228. Cham: Springer, 2018

5 Ichino A, Ikeda T. On the periods of automorphic forms on special orthogonal groups and the Gross-Prasad conjecture. Geom Funct Anal, 2010, 19: 1378-1425

6 Zydor M. Les formules des traces relatives de Jacquet-Rallis grossières. J Reine Angew Math, 2020, 2020: 195-259

7 Beuzart-Plessis R, Liu Y, Zhang W, et al. Isolation of cuspidal spectrum, with application to the Gan-Gross-Prasad conjecture. Ann of Math (2), 2021, 194: 519-584 
8 Beuzart-Plessis R, Chaudouard P-H, Zydor M. The global Gan-Gross-Prasad conjecture for unitary groups: The endoscopic case. arXiv:2007.05601, 2020

9 Zhang W. Fourier transform and the global Gan-Gross-Prasad conjecture for unitary groups. Ann of Math (2), 2014, 180: 971-1049

10 Cai L, Xu B. Isolation of the cuspidal spectrum: The function field case. Sci China Math, 2021, doi: 10.1007/s11425020-1851-y

11 Shalika J A. The multiplicity one theorem for $\mathrm{GL}_{n}$. Ann of Math (2), 1974, 100: 171-193

12 Kaletha T, Minguez A, Shin S W, et al. Endoscopic classification of representations: Inner forms of unitary groups. arXiv:1409.3731, 2014

13 Mok C P. Endoscopic Classification of Representations of Quasi-Split Unitary Groups. Memoirs of the American Mathematical Society, vol. 235. Providence: Amer Math Soc, 2015

14 Zhang W. Automorphic period and the central value of Rankin-Selberg L-function. J Amer Math Soc, 2014, 27: $541-612$

15 Harris R N. The refined Gross-Prasad conjecture for unitary groups. Int Math Res Not IMRN, 2014, 2014: 303-389

16 Beuzart-Plessis R. Plancherel formula for $\mathrm{GL}_{n}(F) \backslash \mathrm{GL}_{n}(E)$ and applications to the Ichino-Ikeda and formal degree conjectures for unitary groups. Invent Math, 2021, 225: 159-297

17 Beuzart-Plessis R. A local trace formula for the Gan-Gross-Prasad conjecture for unitary groups: The archimedean case. Astérisque, 2020, 418: 1-300

18 Xue H. On the global Gan-Gross-Prasad conjecture for unitary groups: Approximating smooth transfer of JacquetRallis. J Reine Angew Math, 2019, 756: 65-100

19 Yun Z. The fundamental lemma of Jacquet and Rallis. With an appendix by Julia Gordon. Duke Math J, 2011, 156: $167-227$

20 Aizenbud A, Gourevitch D, Rallis S, et al. Multiplicity one theorems. Ann of Math (2), 2010, 172: 1407-1434

21 Flicker Y Z. Twisted tensors and Euler products. Bull Soc Math France, 1988, 116: 295-313

22 Jacquet H, Shalika J A. On Euler products and the classification of automorphic representations, I. Amer J Math, 1981, 103: 499-558

23 Zhang W. On arithmetic fundamental lemmas. Invent Math, 2012, 188: 197-252

24 Leslie S, A fundamental lemma for the Hecke algebra: The Jacquet-Rallis case. https://spencerleslie.files.wordpress. com/2020/05/jr-fundamental-lemma-for-hecke-algebra.pdf, 2020

25 Gan W-T, Gross B, Prasad D. Symplectic local root numbers, central critical $L$-values, and restriction problems in the representation theory of classical groups. Astérisque, 2012, 346: 1-109

26 Xue H. Bessel models for real unitary groups: The tempered case. https://www.math.arizona.edu/ xuehang/lggp.pdf, 2019

27 Xue H. Bessel models for unitary groups and Schwartz homology. https://www.math.arizona.edu/ xuehang/lggp_ generic_v1.pdf, 2020

28 Jacquet H, Piatetskii-Shapiro I I, Shalika J A. Rankin-Selberg convolutions. Amer J Math, 1983, 105: 367-464

\title{
The relative trace formula approach for spherical varieties
}

\author{
Li Cai \& Bin Xu
}

Abstract We present a general framework for the relative trace formula approach to the conjecture of Sakellaridis and Venkatesh on period integrals for spherical varieties over global fields. This approach is somehow well-known for experts but it seems lack of literature. Our approach is based on the spectrum isolation technique developed by Beuzart-Plessis et al. (2019) for number fields, which avoids the difficulty from the refined spectral expansion. This technique is also available in the function field case.

Keywords spherical variety, relative trace formula, $L$-function, period integral

MSC(2020) 11F70, 11F72

doi: $10.1360 /$ SSM-2020-0353 